\title{
Das Plancton im Colombo-See auf Ceylon.
}

\author{
Sammelausbeute von A. Borgert, $1904-1905 .{ }^{1}$ ) \\ Von
}

Prof. Dr. C. Apstein in Kiel.

Mit 21 Abbildungen im Text.

Finleitung Seite

Einleitung . . . . . . . . . . . . . . . 202

1. Teil. Die gefundenen Organismen und ihre Periodizität . . 206

I. Algen . . . . . . . . . . . . . . . . . . 206

A. Schizophyceen . . . . . . . . . . . . . 207

B. Flagellaten . . . . . . . . . . . . . . . 207

C. Chlorophyceen . . . . . . . . . . . . . 207

D. Diatomeen . . . . . . . . . . . . . . 208

1) Als erster Beitrag in dieser Reihe erschien W. Michaelsen's Bearbeitung der Oligochäten, über die, zusammen mit anderm Material aus den gleichen Gebieten, im 1. Band der Festschrift für EHLERS berichtet wurde. (Vgl. W. Michaelsen, Die Oligochaeten Deutsch-Ostafrikas, in: Z. wiss. Zool., Vol. 82, 1905.) Michaelsen konnte unter den von mir gesammelten Regenwürmern 12 Species unterscheiden. Es wurde eine neue Gattung (Borgertia) und folgende 7 neuen Arten aufgestellt: Platydrilus armatissimus, Pl. borgerti, Eudriloides platychaetus, Borgertia papillifera, Polytoreutus ehlesi, Pol. usambariensis, Pol. eichelbaumi. - Eine weitere Mitteilung über die Ausbeute meiner Reise brachte E. v. DADAY's Arbeit über den postembryonalen Entwicklungsgang von Caridina wyckii (HICKs) (in Zool. Jahrb., Vol. 24, Anat., 1907), in welcher der Verfasser unter Ausführung der weitern Einzelheiten feststellt, daß der genannte Decapode des Victoria Nyansa, obgleich Süßwasserbewohner, keine abgekürzte, sondern eine vollständige Metamorphose in seiner postembryonalen Entwicklung aufweist. - Vgl. ferner: A. Bongert, Bericht über eine Reise nach Ostafrika und dem Victoria Nyansa nebst Bemerkungen über einen kurzen Aufenthalt auf Ceylon, in: SB. Niederrhein. Ges. Natur- u. Heilkunde Bonn, 1907.

A. BORgERT. 
II. Tiere . . . . . . . . 208

E. Protozoen . . . . . . . . . . . . . . . 208

F. Coelenteraten . . . . . . . . . . . . 209

G. Rotatorien . . . . . . . . . . . . 209

H. Branchiopoden . . . . . . . . . . . . 217

J. Daphniden . . . . . . . . . . . . . . 217

K. Copepoden . . . . . . . . . . 220

L. Ostracoden . . . . . . . . . . . . . 226

M. Insecten . . . . . . . . . . . . . . . . 233

N. Arachnoideen . . . . . . . . . . . . . . . 233

O. Bryozoen . . . . . . . . . . . . . . . . 233

2. Teil. Zusammensetzung des Planctons in den verschiedenen Monaten 233

3. Teil. Fauna des Colombo-Sees . . . . . . . . . . . 237

Tabelle der Fangresultate . . . . . . . . . . . . . . 240

\section{Einleitung.}

Unsere bisherigen Kenntnisse über das Süßwasserplancton tropischer Gegenden rühren von gelegentlichen Fängen her, die Reisende auf ihren Forschungstouren in Seen, Flüssen und Tümpeln machten. Eine zusammenhängende Untersuchung eines größern tropischen Gewässers stand aber noch aus. Die bisherigen Resultate solcher Untersuchungen kamen der Systematik und der Zoogeographie zugute, konnten aber nicht zur Lösung planctonischer, also biologischer Fragen beitragen. Eine Frage, die bisher keine Beantwortung finden konnte, verfolgt mich seit Jahren, ohne daß ich Gelegenheit fand ihr näher zu treten: Haben wir in tropischen Seen ${ }^{1}$ ) eine Periodizität der Organismen? In unsern europäischen Seen, ebenso in denen Nordamerikas ist durch zahlreiche Untersuchungen in den letzten 15 Jahren eine Periodizität in allen Gewässern nachgewiesen worden; Belege dafür brauche ich wohl nicht anzuführen. Über tropische Süßwasserseen war in dieser Beziehung unsere Kenntnis bisher gleich Null. Während der Tiefsee-Expedition untersuchte ich das Plancton des Sinkarah-Sees auf Sumatra, im Colombo-See zu fischen fehlte mir die Gelegenheit, auch reizte mich ein einmaliger Planctonfang weniger. Ich begrüßte es daher mit Freuden als mein Freund Dr. Borgert bei seinem Besuche von Ceylon Herrn Dr. Willey

1) $\mathrm{DaB}$ in kleinen tropischen Gewässern, die austrocknen, eine Periodizität der Organismen herrscht, ist bekannt. Ephippien, Dauereibildung, sind ja Zeichen für die Periodizität und geben Aufschluß, selbst wenn solch ein kleines Gewässer nur Gegenstand einer einmaligen Untersuchung gewesen ist. 
vom Colombo-Museum dafür interessierte, für mich Plancton aus dem Colombo-See zu sammeln und zwar möglichst eine Serie aus verschiedenen Monaten des Jahres. Herr Dr. WiLlex kam dem Wunsche nach und hat, nachdem ich ihm ein Oberflächennetz und Gläser eingesandt hatte, in der Zeit vom 22. Mai bis 28. September 1905 an 11 Tagen Planctonproben in 13 Gläschen gesammelt. Auch an dieser Stelle spreche ich Herrn Dr. Willeer meinen besten Dank für seine Bemühungen aus, sowie Dr. Borgert für seine freundliche Vermittlung. Außerdem erhielt ich ein Gläschen Plancton vom 28. Januar 1905 von Dr. Borgert. Die Fänge sind Horizontalfänge, also nicht quantitativ.

Die Fänge wurden an folgenden Tagen ausgeführt:

1905 Januar 28 (Dr. Borgert).

Mai 22. 29.

Juni 8. 15. 28.

Juli 3. 13. 29.

August 14.

September 8. 28.

außerdem 14. August Bodenmaterial und 28. September „among weeds", also zwischen Uferpflanzen.

Die 12 erstgenannten Fänge habe ich gezählt, im Anhange folgt die Zähltabelle, zu der ich bemerken muß, daß die Organismen innerhalb eines Fanges infolge der zahlenmäßigen Angaben miteinander in Verbindung zu bringen sind, daß aber die einzelnen Fänge nicht miteinander oder nur unter gewissen Bedingungen zu vergleichen sind. Wenn die Serie auch nicht ein ganzes Jahr umfaßt, so zeigen doch die 5 Monate schon, was ich zu wissen wünschte, nämlich - und ich nehme das Hauptresultat meiner Untersuchung voraus - daß eine ausgesprochene Periodizität auch in tropischen Seen zu finden ist, sowohl was das Auftreten und Verschwinden einzelner Arten anbetrifft, als auch ein Ansteigen und Abfallen einzelner Arten nach der Menge, soweit ich bei vorsichtiger Benutzung der qualitativen Fänge darauf Schlüsse ziehen kann.

Von vornherein war die Frage nach der Periodizität nicht zu beantworten. Die Temperatur, das ausschlaggebende Moment in unsern Seen, ist so gleichmäßig in den Tropen, daß hierdurch die Periodizität nicht bedingt sein kann.

Herr Dr. WiLlex übersandte mir die „Administration Reports for 1904 Part 4, Education, Science and Art. Meteorology. Ceylon." 
Auf p. 6 finden sich die Temperaturbeobachtungen, welche für Colombo ein Jahresmittel von $27,27^{\circ} \mathrm{C}^{1}$ ) ergeben, es sind die Mittel der einzelnen Monate nur wenig verschieden: November mit $25,94^{\circ}$ zeigt das Minimum, Mai mit $28,28^{\circ} \mathrm{C}$ das Maximum (siehe Fig. A). Dabei weicht die 1904 gemessene Temperatur vom Mittel (aus 36 Jahren) nur so wenig ab, daß ich die normalen Mittel als für 1905 geltend direkt annehmen kann. Die Temperatur - wie am deutlichsten Fig. A zeigt - hat nur ganz minimale Schwankungen im Laufe des Jahres. Daher ist nicht anzunehmen, daß diese kleinen Unterschiede einen besondern Einfluß auf das Plancton ausüben.

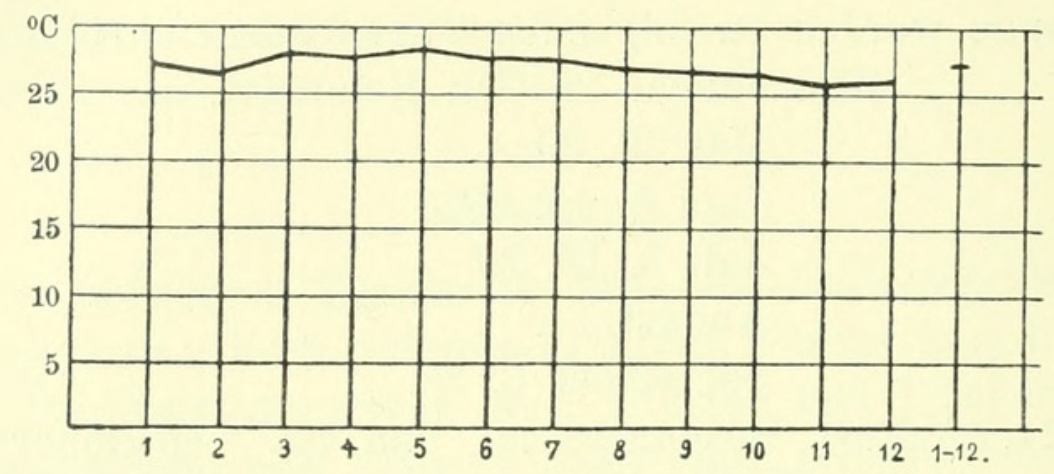

Fig. A.

Temperaturkurve.

$1 \mathrm{~mm}=1^{0} \mathrm{C}$ normal (Mittel). Abscisse: Monate. Ordinate: Temperatur von $5 \mathrm{zu} 5^{\circ} \mathrm{C}$.

Das andere Moment, das in Betracht kommen könnte, ist der Wechsel von Regen und Trockenzeit. In West-Ceylon haben wir ich verdanke die Mitteilung darüber Herrn Prof. KrümmelL - zwei Regenzeiten. Aus den genannten Administration Reports geht dieses Factum auch deutlich hervor (p. F 10). Die beifolgende Kurve über die Regenhöhe ist nach dem Mittel aus 35 Beobachtungsjahren gezeichnet (Fig B). Punktiert ist die mir von Herrn Prof. KRüMmelu mitgeteilte für 1905 beobachtete Regenhöhe (nach Sruons, in: Meteorol. Magazine, 1905/6, No. 477-482) eingezeichnet. Das Minimum 15 mm fand sich erst im August, im übrigen bleiben in den uns interessierenden Monaten die Regenmengen hinter den normalen zurück: April bis September 935,8 mm, dagegen normal: 1514,9 mm.

1) Die Angaben im Original sind in Fahrenheit, die Regenmengen in "inches", beide Maße sind umgerechnet. 
Fig. B zeigt deutlich, daß ein starker Wechsel in der Regenmenge vorhanden ist. Damit hängt die starke Schwankung in der Tiefe des Colombo-Sees zusammen, wie sie tab. 1 in dem Administration Reports zeigt und ferner die verschiedene Zufuhr von Nahrung in den See. Der Regen befördert viele Stoffe vom Ufer in das Wasser, laugt das Land aus, sodaß bei größerer Regenmenge auch die Organismen im See direkt oder indirekt eine größere Nahrungsmenge zur Verfügung haben.

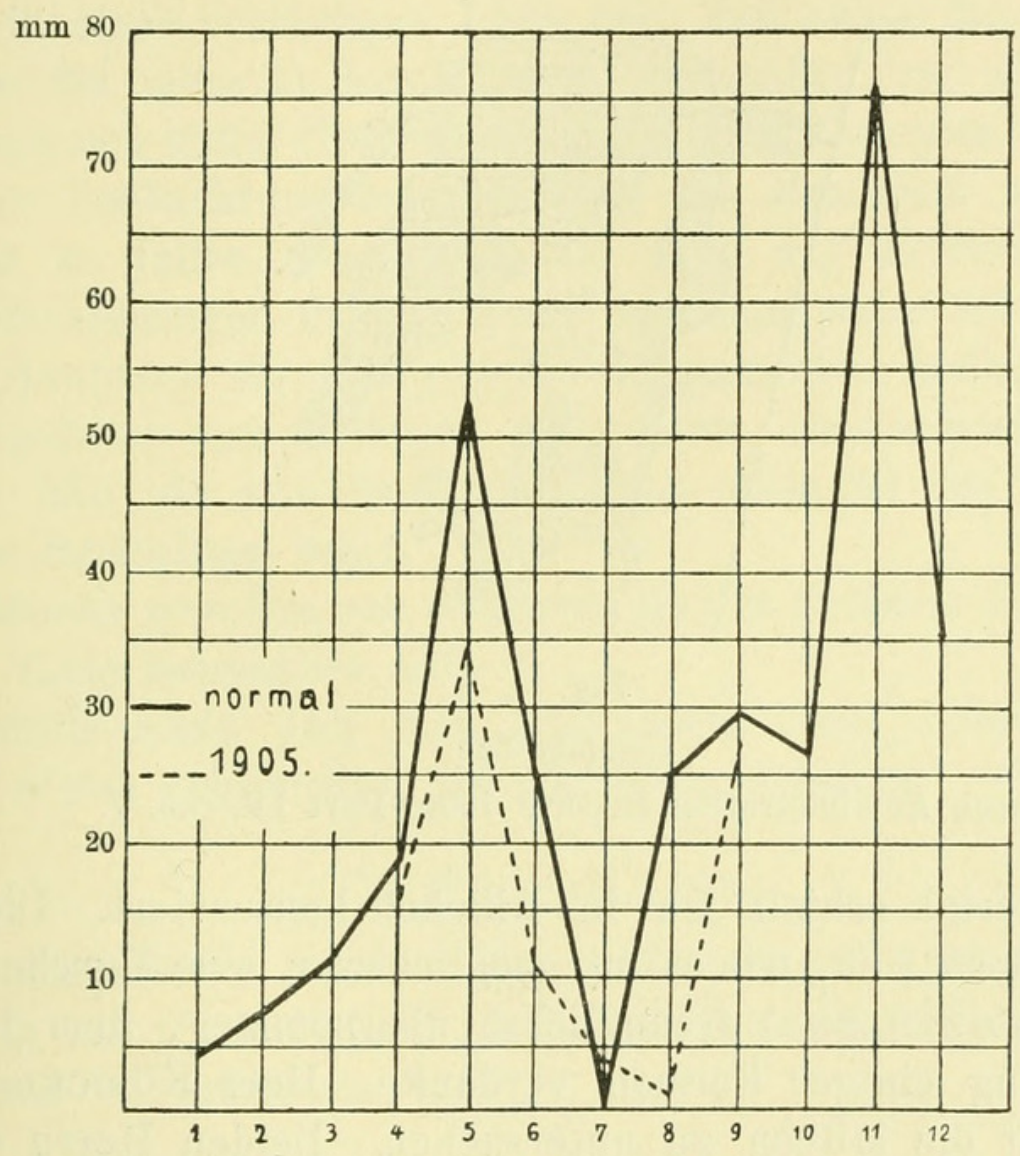

Fig. B.

Regenhöhe.

$1 \mathrm{~mm}=10 \mathrm{~mm}$ Regen. Abscisse: Monate. Ordinate: Regenhöhe von $50 \mathrm{zu} 50 \mathrm{~mm}$.

Der Colombo-See (Fig. C) hat eine Oberfläche von 1,5 qkm. Herr Prof. Krüмmelu hatte die Freundlichkeit, den See nach tab. 7 der Administration Reports für mich mit dem Polarplanimeter auszumessen, wofür ebenso wie für die andern Notizen ich ihm auch hier meinen besten Dank sage. Allerdings wird die Größe des Sees wohl großen Schwankungen unterworfen sein, da nach tab. 1 der genannten Reports seine Tiefe sehr wechselt und damit das Wasser 
vom Ufer zurücktreten muß. So betrug - wenn ich tab. 1 recht verstehe - am 5. Juli die Tiefe am Pegel nur 2 Zoll, während sie am 10. Januar mit 2 Fuß 7 Zoll als Maximum des Jahres 1904 gemessen war. Der See ist „during the dry season of the North East Monsoon [also in unserm Winter] choked with weeds", schreibt mir Dr. Willey. Daher auch aus dieser Zeit keine Planctonfänge stammen.

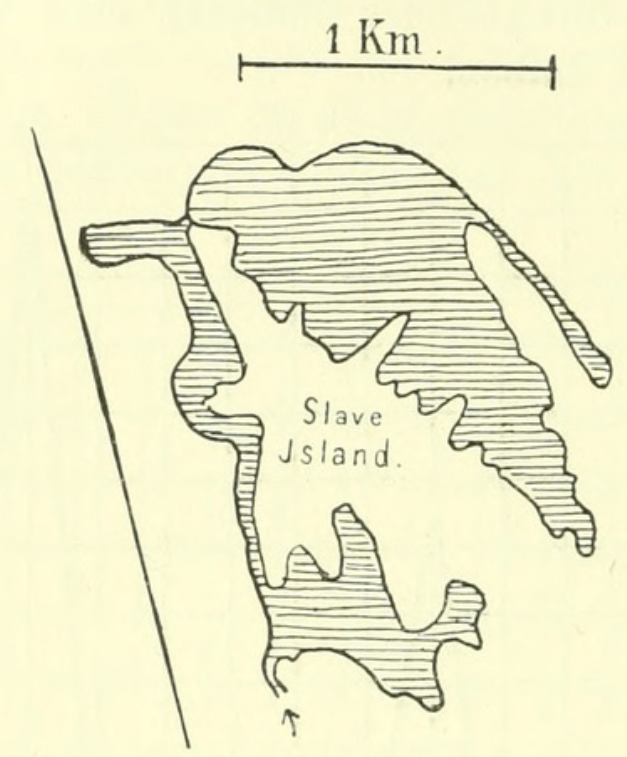

Fig. C.

Colombo-See

nach Administration Reports 1904, Part IV, tab. 7.

Das Material erhielt ich in Alkohol konserviert. Die Algen bestimmte ich so weit, wie es mir möglich war, eine Durcharbeitung hat Herr Lemmenmann ${ }^{1}$ ) freundlichst übernommen, dem ich auch die Bestimmung einiger Formen verdanke. Herr Könıcke hat die Freundlichkeit die Milben zu untersuchen. Beiden Herrn sage ich für ihre Hilfe meinen besten Dank.

\section{Teil.}

\section{Die gefundenen Organismen und ihre Periodizität.}

\section{Algen.}

Unter den Algen spielten nur 2 Arten eine größere Rolle, Clathrocystis und Melosira, alle andern waren nur kürzere Zeit und in

1) Die Arbeit soll unter dem Titel „Beiträge zur Kenntnis der Planktonalgen. XXII. Phytoplankton von Ceylon" erscheinen. 
nicht zu großen Mengen vorhanden. In meiner Tabelle (siehe Schluß) habe ich nur 9 Arten aufgeführt; eine genauere Durchmusterung, die Herr Lemmermann freundlichst übernommen hat, wird noch mancherlei seltenere Arten zutage fördern. Ich konnte mich daher auf das Wichtigste beschränken.

A. Schizophyceen.

Clathrocystis aeruginosa (KüTz) Henfr. ${ }^{1}$ ) Herr Lemmermann teilt mir in einer vorläufigen Liste mit, daß außer dieser Art auch Microcystis flos aquae in dem Material vorhanden ist; ich habe wohl beide zusammen unter Clathrocystis notiert. Clathrocystis war während der ganzen Beobachtungszeit häufiger, das Maximum schien in den September zu fallen, also dann, als nach der Trockenzeit wieder größere Regenmengen fielen. Auch bei uns habe ich die gleiche Zeit als Hauptzeit für Clathrocystis (3) gefunden, bedingt durch die hohe Temperatur und vielleicht auch durch die sommerlichen Regen. Vielleicht hält sie sich in Ceylon auch während des Winters, wie der große Januarfang zeigt.

*Anahaena spiroides var. contracta trat in größerer Zahl nur Ende Juli und Ende September auf.

Ebenfalls Ende Juli war eine noch nicht näher bestimmte Oscillarie etwas zahlreicher, die ich sonst vermißte.

B. Flagellaten.

Von Flagellaten wurden öfter Colacium auf Cyclops beobachtet, namentlich am 15. Juni und 13. Juli. Es handelt sich um *C. vesiculosum und $* C$. arbuscula.

\section{Chlorophyceen.}

*Volvox aureus EнBg. fand ich in einem schönen Exemplar am 14. August; also zur Zeit der größten Trockenheit. Kleinere, noch nicht näher bestimmte Volvocaceen fanden sich häufiger vom 28. Juni bis 14. August, also auch zur Zeit des geringsten Niederschlags.

* Pediastrum duplex var reticulatum LAG. fand sich im Januar, dann von Ende Juni bis Ende Juli, aber nie in vorherrschender Stellung. *Closterium lumula var. coloratum KLEBs. fand ich zum ersten Male

1) Die mit einem * versehenen Arten sind von Herrn Lemmermann bestimmt oder wenigstens meine Gattungsbestimmungen durch ihn mit Art und Varietätszusätzen versehen worden. 
am 28. Juni, dann erst wieder am 29. Juli, von wo an es an Zahl zuzunehmen schien, sodaß ich am 8. September schon 500 notieren konnte, am 28. September fehlte es aber schon wieder.

\section{Di a tom een.}

* Melosira gramulata forma war vom 15. Juni bis 8. September vorhanden, also während der trockensten Zeit des Jahres (Fig. B). Am 28. Juni müssen wir das Maximum annehmen, da ich an diesem Tage über 11 Millionen Fäden fand. Selbst wenn die Zahl für diesen Tag mehrmals zu hoch ist, oder alle andern Zahlen mehrmals zu niedrig, so wird keine an die Zahl des 28. Juni heranreichen.

\section{Tiere. ${ }^{1}$ )}

\section{E. Protozoen.}

Freischwimmend wurde nur 1 Art, Coleps hirtus Eнвg. angetroffen. Er kam verstreut vor, häufiger am 29. Mai und 28. Juni aber auch in den Zwischenzeiten, vom Mai bis Ende Juli, dann fehlte er.

Passivpelagisch fanden sich Vorticellen auf Cyclops am 8. und 15. Juni. Dann ein Organismus, den ich für Trichodina halte. Letztere saßen stets auf Ostracoden, sowohl auf leeren Schalen, als auch auf denen noch lebender Tiere. Namentlich zahlreich waren sie am 14. August, von welchem Tag ich einen Fang hatte, bei dem das Netz auch Bodenmaterial gefischt hatte.

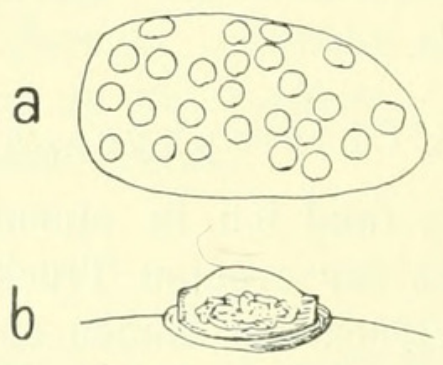

Fig. D.

Trichodina auf Ostracodenschalen.

a $33: 1$. b einzelne Trichodina. $180: 1$.

Der Durchmesser der Trichodina ist $70 \mu$. Ein flacher Rand liegt der Ortracodenschale auf, innerhalb dessen sich ein niedriger

1) Für einschlägige Literatur bin ich den Herren Prof. v. DADAY, BARROIS und KönicKe zu großem Dank verpflichtet. 
Zylinder erhebt, sodaß das ganze den Eindruck eines flachen Hutes macht.

\section{F. Cölenteraten.}

Hydra fand sich in 2 Exemplaren am 28. September „among weeds", war also von Pflanzen abgerissen, auf denen sie gesessen hatte.

\section{G. Rot atorien.}

Eine große Rolle spielen die Rädertiere. Während bisher nur eine Art Rotifer vulgaris durch v. DADAY (4) für den Colombo-See nachgewiesen war, habe ich deren 13 gefunden.

Asplanchna brightwelli Gosse. Der Kiefer (Fig. E) ähnelt am meisten dem von Hudson u. Gosse (7, tab. 12, fig. 1b) abgebildeten, jedoch hat der Kiefer nicht 2 Spitzen, sondern 1 Spitze und daneben 1 zahnartige Platte, sodaß er bei schwacher Vergrößerung 2spitzig. erscheint. Mit der Varietät ceylanica von v. Daday (4) stimmt er nicht überein, da der Mittelzahn ganz anders ist, ebenso wie der übrige Bau des Kiefers. v. DaDAY fand die Varietät in den Sümpfen am KalawewaSee auf Ceylon.

Meine Exemplare hatten auch ein wurstförmiges Ovarium. Die Art fand sich fast in allen Fängen, am zahlreichsten am 15. Juni, dann Juli und August, also wieder in der Trockenzeit. Auch im Januar waren zahlreiche Individuen vorhanden.

Zeitweise wurden sehr viel Junge produziert, so betrug das Verhältnis zwischen

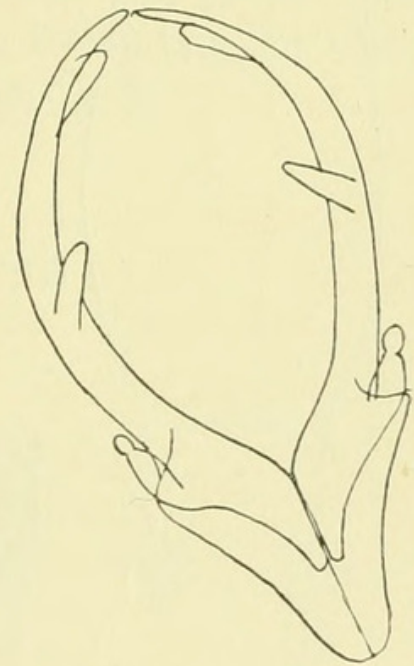

Fig. E.

Kiefer von Asplanchna brightwelli. $300: 1$.

Erwachsenen und Jungen am 3. Juli $3: 2$, am 14. August 5: 1, und am 28. Januar $7: 3$.

Synchaeta sp. Im Mai, Juni bis Juli war eine Synchaeta, die der starken Kontraktion wegen von mir nicht näher bestimmt werden konnte, häufiger. Namentlich am 8. Juni fanden sich zahlreichere Individuen in dem nicht allzugroßen Fange. Eier beobachtete ich namentlich im Mai, auf jedes 2.-3. Individuum kam 1 Ei. 
Triarthra longiseta EнвG. Diese Art trat schon in den Maifängen auf, war aber nicht häufiger. Erst Ende Juni erschien sie massenhaft und fand sich dann in allen Fängen bis Ende September d. h. soweit die Untersuchung reicht, auch im Januar fand ich zahlreiche Exemplare. Während dieser Zeit wurden auch zahlreich Eier erzeugt. Es kamen auf 100 Individuen am 28. Juni 66 Eier, am 13. Juli 24, am 29. Juli 10, am 14. August 40 und am 28. September 50 Eier. Die Fruchtbarkeit hatte bis zum 29. Juli abgenommen, stieg dann aber wieder rasch an.

Polyarthra platyptera EHBG. Seltener war dieses Rotator. Es fand sich hauptsächlich während des Juni, einmal trat es noch im September auf.

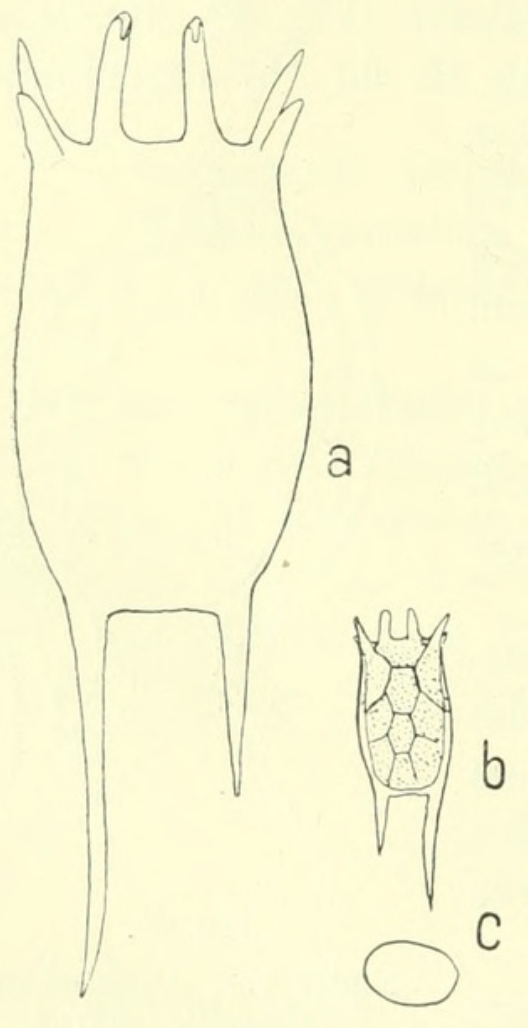

Fig. F.

Anuraea valga var. tropica.

a vom Bauche. 300:1.

b vom Rücken. 100:1.

c Ei. $100: 1$.
Mastigocerca sp. war nur einmal in geringer Zahl in den Fängen vorhanden.

Anuraea valga EHBG. var. tropica ${ }^{1}$ ) Apst. (Fig. F). Ohne die hintern Hörner ist diese Varietät 0,169 mm lang, die beiden Hinterhörner sind ungleich lang, das eine $0,088 \mathrm{~mm}$, das andere $0,038 \mathrm{~mm}$, also größer als A. valga EнвG. Die beiden Mittelhörner sind an der Spitze bauchwärts gebogen, die Täfelung und Struktur des Panzers ist wie bei valga.

Das Ei ist oval, die große Achse mißt $0,068 \mathrm{~mm}$.

Vom 29. Mai an fand sich diese Art zahlreich im Plancton. Ihre Hauptzeit scheint vor der Zeit der größten Trockenheit, also dann, wenn der See am wenigsten Wasser führt - wohl 1905 August - zu liegen, wir finden da Fänge mit 10 und 14000 Exemplaren.

1) Die gleiche Varietät fand ich im Sinkarah-See auf Sumatra im Januar 1899. 
Im Mai bis Juni ist die Fruchtbarkeit am größten, wir finden auf 2-4 Individuen ein Ei, während später erst ein Ei auf 7-17 Individuen kommt.

Brachionus amphiceras EHBg. var. borgerti Apst. (Fig. G). Meine Exemplare glaube ich als Varietät zu der EHREnBerg'schen Art amphiceras (9, tab. 63, fig. 2) stellen zu müssen, da mannigfache Abweichungen existieren. Das Vorderende bei der Varietät und Stammart ist ähnlich, jedoch sind bei den ceylonischen Exemplaren die Fortsätze länger, und die mittlern tragen an ihrer Basis noch einen zahnartigen Fortsatz. Der Panzer ist nach hinten verbreitert und trägt da, wo er sich wieder verschmälert, die großen seitlichen Fortsätze. Die kleinen Fortsätze neben dem Fuß sind ähnlich, wie Ehrenberg sie abbildet. Die Länge des Panzers ohne Fuß ist $0,375 \mathrm{~mm}$, der $\mathrm{FuB} 0,169 \mathrm{~mm}$ lang. Von Eiern kamen 2 Sorten vor, beide dünnschalig. Das größere Ei war oval, die große Achse 0,188 mm lang, das kleinere Ei mehr rundlich, 0,082 mm im Durchmesser (Sommer- oder Weibchen- und Männcheneier?).
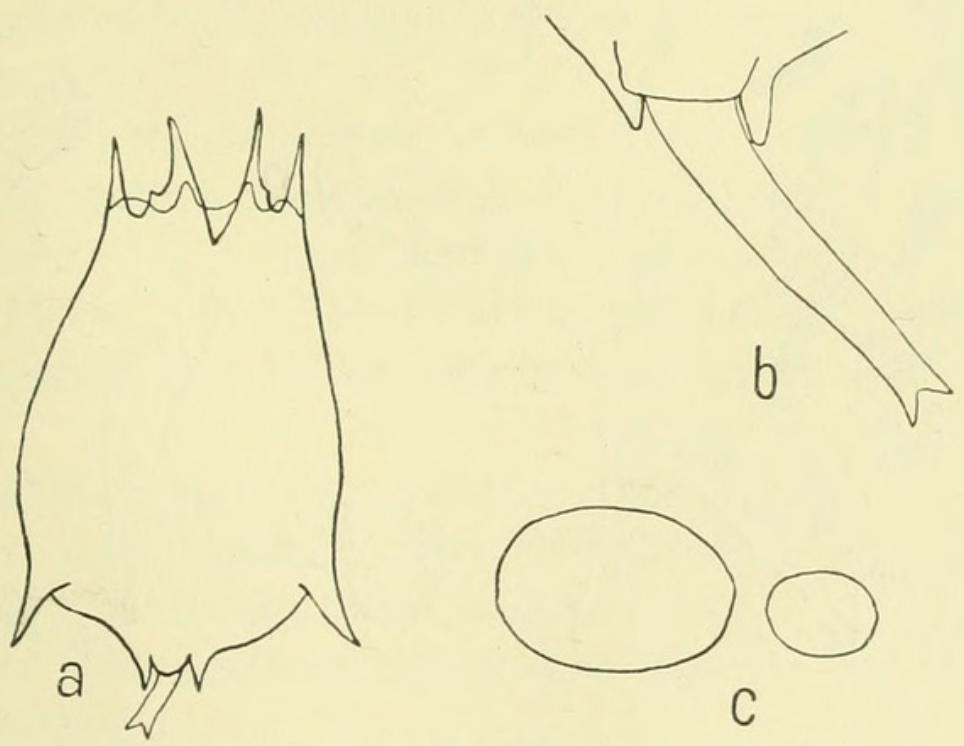

Fig. G.

Brachionus amphiceras var. borgerti.

a $100: 1$. b Fuß. 180:1. c Eier. 100:1.

Während der ganzen Zeit fand sich dieses schöne, große Rädertier, aber in wechselnder Häufigkeit. Leider lassen die qualitativen Fänge keine Schlüsse auf die Periodizität zu, nur soviel scheint mir sicher, daß am 28. September die Individuenzahl besonders hoch 
war, also mit Beginn der Regenzeit. Während der ganzen Beobachtungszeit fanden sich die großen Eier (Weibcheneier c), und meist war ihr Verhältnis zu den Müttern recht günstig. Mehrmals kommt auf jedes Individuum im Durchschnitt ein $\mathrm{Ei}$, die ungünstigsten Zahlen finden sich am 22. Mai 1:7 und am 14. August 1:37, sonst höchstens 1:3. Die Weibchen trugen 1-2 Eier. Die kleinern Eier (d) - vielleicht Männcheneier - wurden nur am 28. September gefunden, auf jedes 17. Weibchen kam im Mittel ein kleines Ei. Die Zahl der Eier, die ein Weibchen trug, schwankt zwischen 1 und 10 .

Namentlich am 15. Juni und 28. September fand ich in dem genannten Brachionus einen Parasiten (Fig. H), der meist in zahlreichen Exemplaren vorhanden war. Die Rädertiere waren dabei wohl erhalten. Ich konnte über die Natur des Parasiten keine
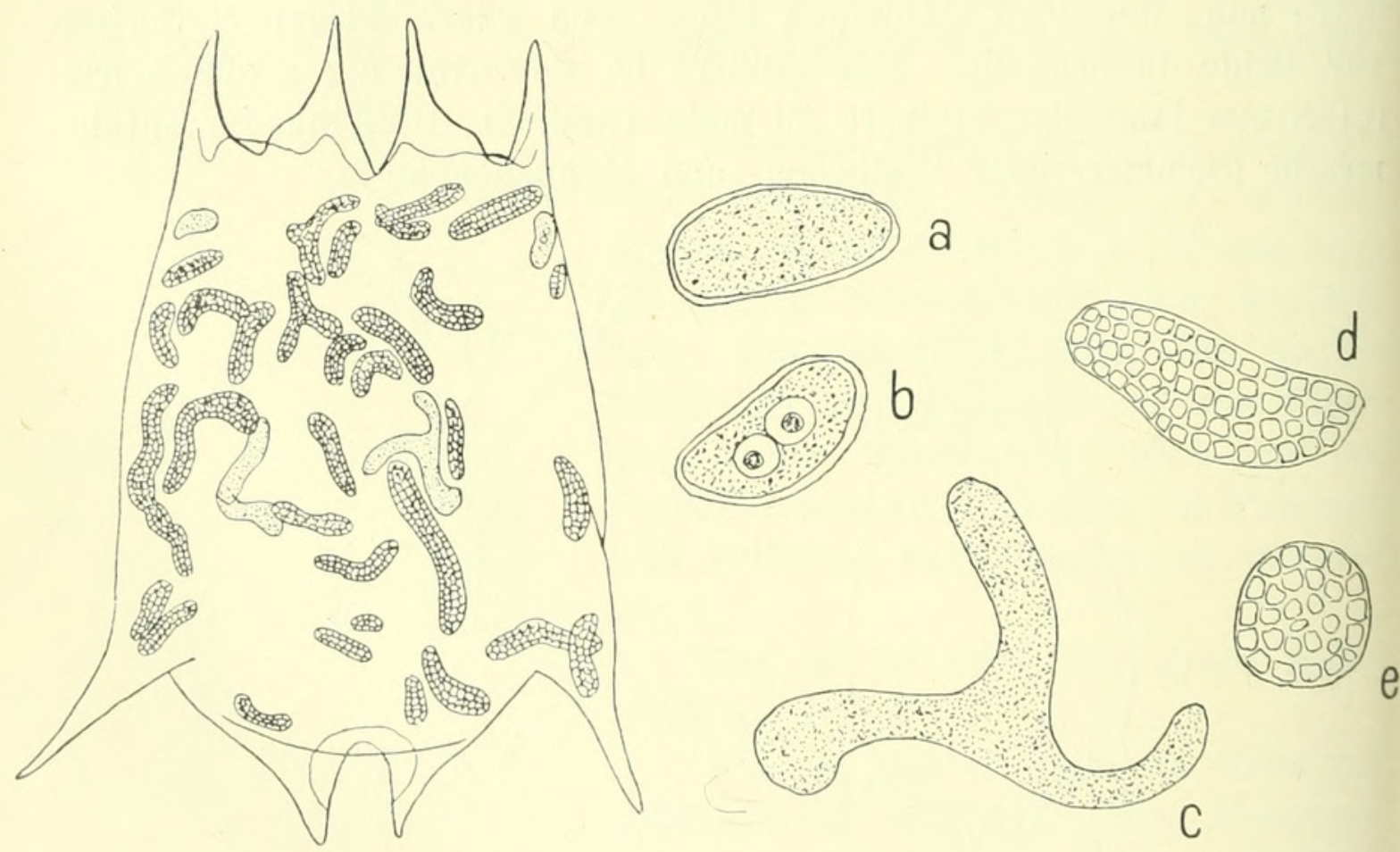

Fig. H.

Brachionus mit Parasiten. $270: 1$.

a kernloser(?), b mit Kernen versehener Parasit. c verzweigtes Exemplar.

d großzelliges Exemplar, Längsschnitt, und e im Querschnitt. Alles 470:1.

(Ölimmersion).

Klarheit erhalten, trotzdem ich denselben auch geschnitten habe, will aber doch Abbildungen geben, damit er event. an lebendem Material weiter studiert werden kann. Bertram (16) hat wohl den- 
selben Parasiten studiert, und bei LabBé (17 p. 111) finde ich ihn unter dem Namen Plistophora sp. angeführt. Ich folge dem ausgezeichneten Forscher über die Sporozoen und bezeichne deshalb auch den von mir gefundenen Parasiten als Plistophora. Die Parasiten waren eiförmig (Fig. Ha, b), wurstförmig (Fig. H) oder verzweigt (Fig. Hc). An den eiförmigen Individuen (Fig. Ha) konnte man feinkörniges Plasma erkennen, das von einer feinen Membran umgeben war. Die Individuen hatten Kerne (Fig. Hb), die in einem größern Saftraum lagen, andere ließen die Kerne vermissen, vielleicht waren sie in kleine Stücke zerfallen und durch das Plasma zerstreut.

Ebensolch feiner Inhalt ohne Kerne ließ sich bei den größern wurstförmigen und verzweigten Parasiten erkennen (Fig. H c). Bei der Mehrzahl der Exemplare aber war der Körper aus größern Zellen zusammengesetzt, in denen ich keine Kerne erkennen konnte, auch keine weitern Differenzierungen, wie ich auf Schnitten sah (Fig. $\mathrm{Hd}$, e). Ehrenberg (9, p. 513, tab. 63, fig. 9) erwähnt bei Brachionus mülleri „ein Individuum, welches während seines Lebens mit vielen lebenden Monaden, wahren Entozoën, erfüllt war". Aus der Figur läßt sich aber nichts sicheres erkennen.

Brachionus pala Eнвg. var. willeyi Apst. (Fig. J). Dieses Rädertier war dem vorigen sehr ähnlich, unterschied sich von ihm hauptsächlich durch das Fehlen der hintern seitlichen Fortsätze. Auch die Fortsätze neben der Austrittsstelle des Fußes sind anders ausgebildet.

Am nächsten steht die Varietät dem $\mathrm{Br}$. pala EHвG., jedoch tragen die Mittelhörner am Vorderende noch deutliche seitliche Zähne, dann ist der Einschnitt zwischen den Mittelhörnern sehr tief.

Die Länge beträgt $0,4 \mathrm{~mm}$, während Ehrenberg für pala $0,27-0,55 \mathrm{~mm}$ angibt. Eier fanden sich in 2 Sorten, erstens größere runde Eier von 0,16 mm Durchmesser, dann kleinere ovale, deren große Achse $0,093 \mathrm{~mm}$ maß. Ehrenberg (9) zeichnet für $B$. pala ein großes, ovales oder viele kleine ovale Eier.

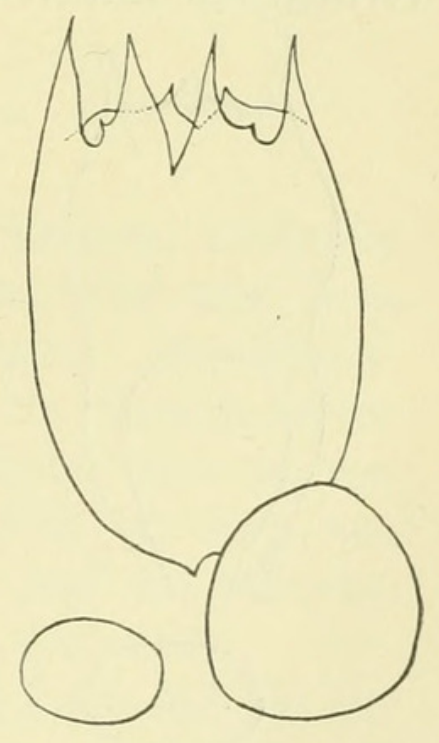

Fig. J.

Brachionus pala var. willeyi mit Ei. $100: 1$. 15 
Unser Rädertier trat im Juni und nur einmal im September in kleinerer Zahl auf. Stets hatten die Tiere zahlreichere Eier produziert. Das ungünstigste Verhältnis von Ei zu der Mutter war 1:4, meist viel günstiger. Am 1ə̌. Juni fanden sich zahlreicher die kleinen (Männchen-?)Eier. Auf 100 Weibchen kamen 16 Eier, im September auf 100 Weibchen 5. Die Weibchen trugen 1-3 große oder 1-4 kleine Eier, wobei zu bedenken ist, daß Eier abgefallen sein können und von mir nicht als zu dieser Art gehörig erkannt sind. Natürlich habe ich stets auch die freiliegenden Eier, nicht nur die an den Weibchen klebenden Eier berücksichtigt, sofern ich sie zu der Art gehörig erkennen konnte.

Brachionus rubens EHBg. Das von mir mit diesem Namen belegte Rädertier entspricht gut der fig. 5 auf tab. 27 bei Hudson u. Gosse (7). Nur eine kurze Lebensdauer scheint dieser Art im Colombo-See vergönnt zu sein, die ersten Exemplare fand ich am 8. Juni, die letzten am 3. Juli. Vereinzelte Exemplare bleiben wohl am Leben, wie der Fund eines Tieres am 8. September zeigt. Die Eiproduktion war in der Mitte der Vegetationsperiode am größten, zuerst 1:50, dann 1:3,1:2 und schließlich 1:62. Zu Beginn der Trockenzeit war das Rädertier wieder verschwunden.

Brachionus forficula Wienz. var. levis Apst. (Fig. K). Das mir vorliegende Rädertier erschien auf den ersten Blick wie B. forficula

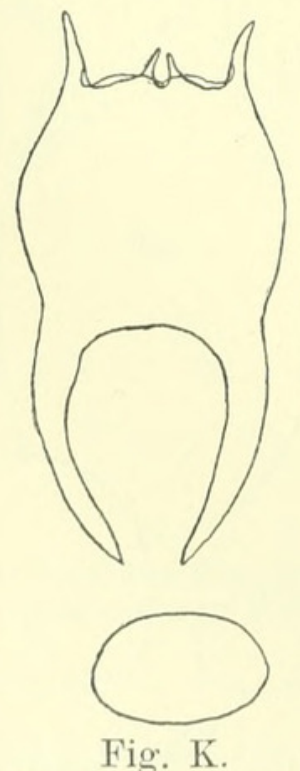

Brachionus forficula var. levis mit Ei. 180: 1 . Wierz. (8), weicht von diesem aber doch so $a b$, daß ich gezwungen bin eine neue Varietät zu bilden. Der Panzer ist glatt, die vordern Seitenhörner sind viel länger als die mittlern, diese konvergieren nach der Spitze zu. Die Hinterhörner haben keine Ausbuchtungen an der Basis wie die Stammform. Der Panzer hat keine Felderung und ist glatt nicht gekörnt.

Die Länge beträgt $0,226 \mathrm{~mm}$, ist also etwas größer als die der Stammform. Ich fand nur eine Art von Eiern, die oval sind, die große Achse mißt $0,065 \mathrm{~mm}$. Diese Art fand ich nur im Mai und September und Januar, also in der Regenzeit. Meist trug jedes zweite Weibchen ein Ei, nur Ende September kam ein Ei auf 4 Weibchen und im Januar ein Ei auf 6,5 Weibchen. 
Brachionus falcatus ZACH. var. $\beta$ Weber (Fig. L). Dieses schöne Rädertier ist von Weber (19, p. 219) nach Material aus Java beschrieben worden.

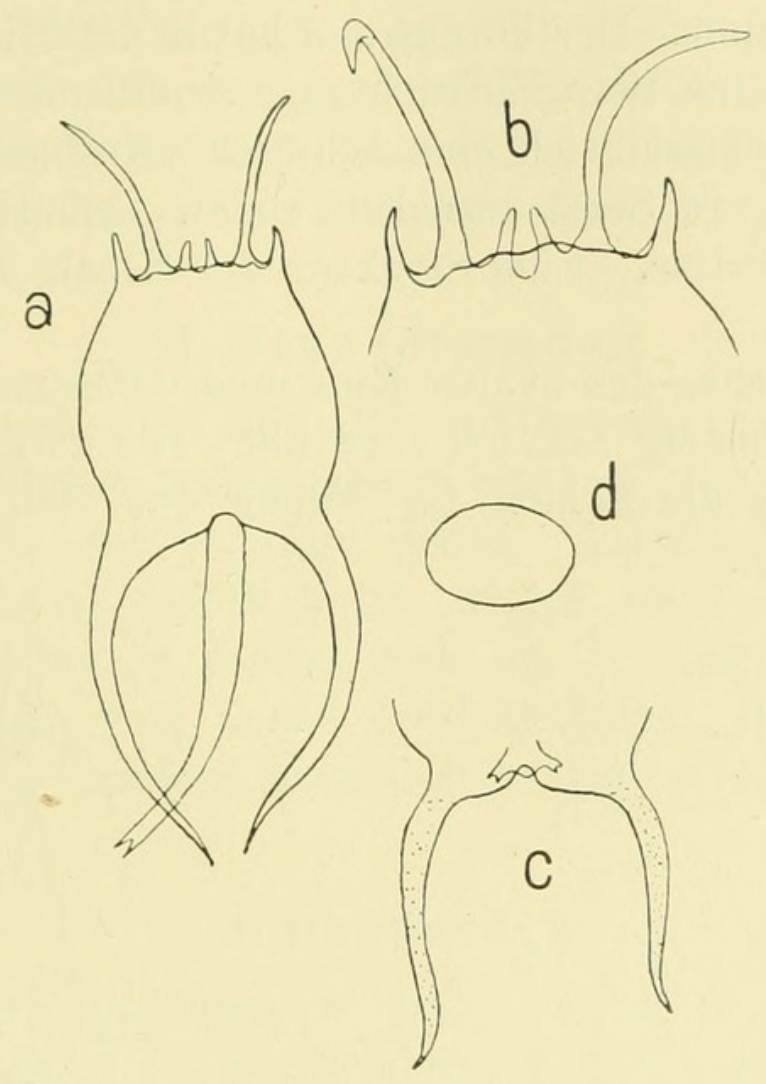

Fig. L.

Brachionus falcatus var. $\beta$.

a $100: 1$. b Vorderende. $180: 1$. c Hinterende. $100 ; 1$. d Fi. $100: 1$.

Am Vorderrande finden sich 6 Hörner, die mittlern, kleinsten konvergieren nach der Spitze zu, das 2. und 5. Horn sind sehr lang, nach auswärts und nach der Bauchseite zu gebogen. Die Hinterhörner sind kräftig, nach innen gebogen oder die Spitzen etwas nach außen. Der Panzer ist glatt, höchstens findet sich an den Hinterhörnern eine ganz feine Körnelung. Der Fuß ist lang und kräftig. Die Länge beträgt $0,55 \mathrm{~mm}$. Von Eiern fand ich nur eine Sorte, die oval war und deren große Achse 0,094 $\mathrm{mm}$ maß.

Die Art fand sich im Mai und Juni, dann von Ende Juli bis Anfang September. Die größte Zahl fand ich im August zur Zeit der größten Trockenheit. Anfang Juni war Br.falcatus var. $\beta$ schon häufig, verschwand aber wieder um Ende Juli und August wieder häufig aufzutreten. Während der Hauptzeit war die Fruchtbarkeit groß, 
jedes 2.-4. Weibchen trug ein Ei. Anfang September hatten die 100 Weibchen nur noch 2 Eier produziert.

Brachionus caudatus Barr. et DaDAY (Fig. M). Die Länge dieser Art beträgt $0,179 \mathrm{~mm}$. Der Vorderrand hat in der Mitte 2 kurze Fortsätze, die durch eine tiefe Einbuchtung voneinander getrennt sind. Nach der Seite schließt sich eine schwache Ausbuchtung an, die in einem etwas längern Seitenhörnchen endet. Am Hinterende finden sich 2 lange Fortsätze. Eine Struktur der Schale konnte ich nicht sehen.

Die große Achse des ovalen Eies maß 0,094 mm. Br. caudatus trat sehr unregelmäßig auf, ich fand die Art im Juni, Juli und September, ohne daß die Zahlen für irgend eine Zeit eine besondere Häufigkeit zeigten.

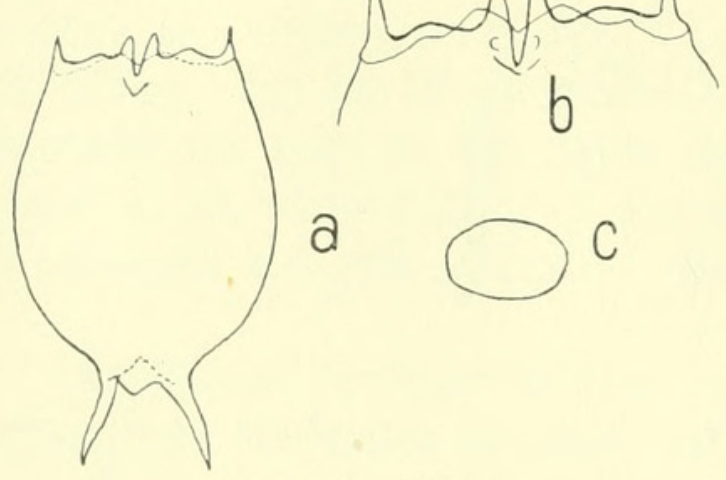

Fig. M.

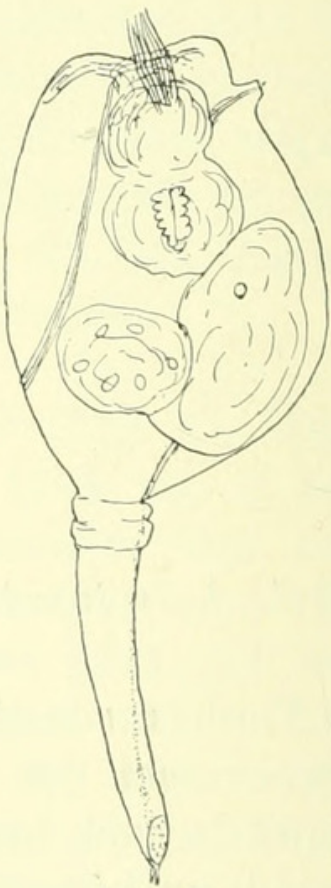

Fig. N.

Fig. M. Brachionus caudatus. a $100: 1$. b Vorderraud. 180:1. c Ei. 100:1.

Fig. N. Notops macrurus von der Seite und Zehen vom Rücken. 180: 1 .

Notops macrurus Barr. et Daday (Fig. N). Meine Exemplare waren etwas kleiner als $v$. DADAY (8) für die seinen aus dem Albollonia Göl angibt, sie maßen nur $0,36 \mathrm{~mm}$, wovon auf den Fuß $0,159 \mathrm{~mm}$ entfielen. Bei der Seitenlage war der Taster sehr gut sichtbar.

Die Art trat nur während der Trockenzeit, also von Ende Juli bis Anfang September, auf, war in dieser Zeit aber in größerer Zahl vorhanden und produzierte reichlich Eier. 
Pedalion mirum Huds. v. DadaY (8) hat dieses Rädertier in Kleinasien gefunden, bezeichnet es aber als selten. Ich konnte dasselbe für Ceylon nachweisen und muß es als zeitweise häufig bezeichnen. Am 29. Mai sah ich die ersten Individuen, im Juni nahm die Zahl sehr zu, um Mitte Juli ganz abzunehmen. Im August und September war die Art nur noch selten. Während der Hauptzeit, also kurz vor der Trockenzeit, kam ungefähr auf jedes 4 . Weibchen 1 Ei. Ganz besonders viele Individuen fand ich im Januarfang.

H. Branchiopoden.

Cyclestheria hislopi (BAIRD). Diese interessante Art ist schon von Brady (5) für Colombo nachgewiesen worden. Sie gehört nicht zum Plancton, sondern lebt auf dem Boden. Am 29. Juli, als das Netz etwas Bodenmaterial gefaßt hatte, waren im Fange 4 Exemplare. Zahlreichere Individuen fanden sich am 14. August, als ein Fang eine größere Probe vom Boden gefischt hatte. Die Art muß sehr häufig sein, da so zahlreiche Exemplare in den verhältnismäßig kleinen Proben waren.

\section{J. Daphniden.}

Diaphanosoma singalense v. DaDAY (Fig. 0). Diese Art hat v. Daday von Ceylon (4) beschrieben, wo sie von ihm für mehrere Fundorte angegeben wird; für den Colombo-See ist sie neu. Ich fand diese Daphnide während der ganzen Beobachtungszeit, hauptsächlich aber im Juni und Juli, also in der Trockenzeit, dann aber auch
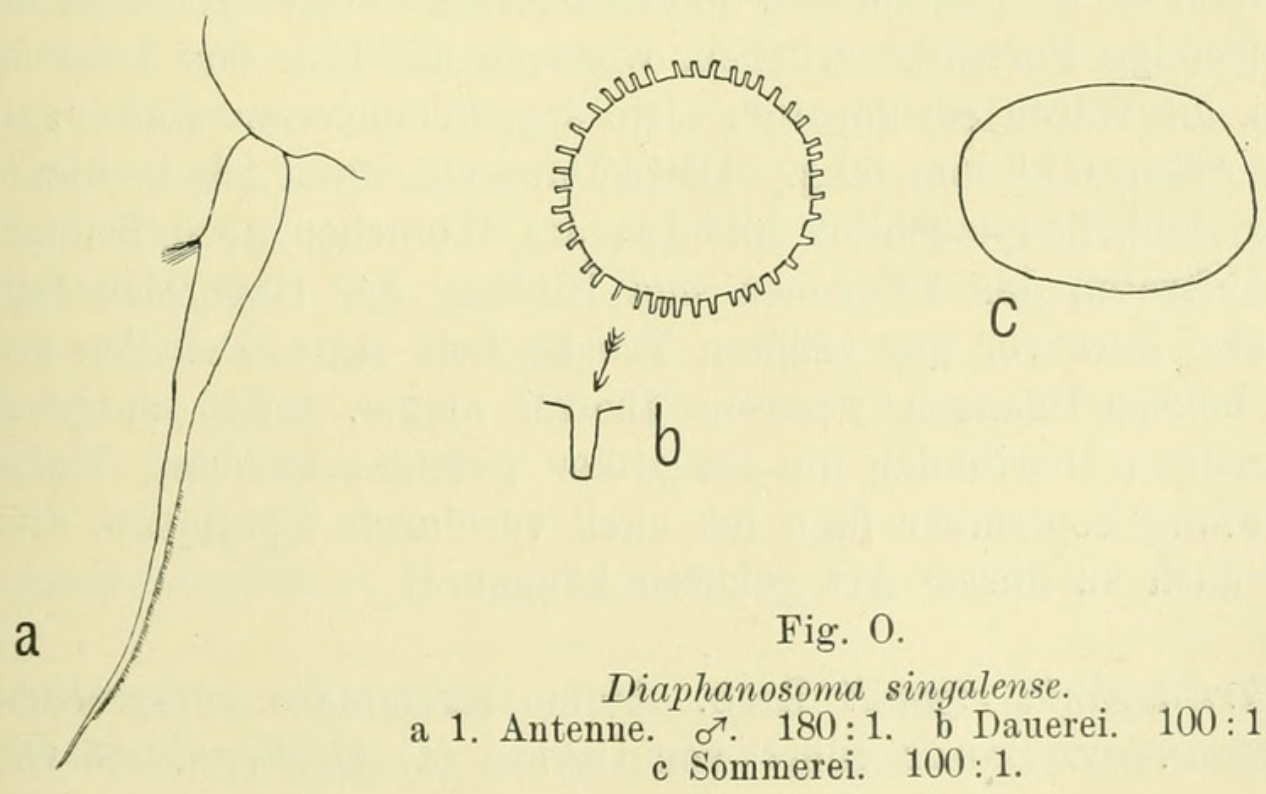

Fig. 0.

Diaphanosoma singalense.

a 1. Antenne. ơ. $180: 1$. b Dauerei. $100: 1$.

c Sommerei. $100: 1$. 
wieder im Januar, also auch in der trockensten Zeit des Winters. Im Mai waren in den Fängen nur wenige Exemplare, von denen einige Eier (Fig. Oc) besaßen. Im Juni nahm die Zahl gewaltig zu und ebenso die Eiproduktion. Das Verhältnis der Eizahl zu der der Weibchen betrug in der Hauptzeit $1: 2^{1 / 2}$ bis $1: 20$. Neben den Eiern fanden sich aber noch immer zahlreich ganz junge Tiere, rechnet man diese mit den Eiern zusammen, so erhalten wir vom 8. Juni bis 29. Juli:

$\begin{array}{lrrrrrr}\text { Weibchen } & 5750 & 1250 & 1250 & 5000 & 600 & 2550 \\ \text { Eier und Junge } & 1100 & 1350 & 900 & 14800 & 1400 & 2000 .\end{array}$

Danach wird die Verhältniszahl $1:{ }^{1 / 3}$ bis $1: \check{5}$. Namentlich zeichnet sich der 3. Juli durch die große Zahl der Jungen aus. Auf 5000 Weibchen kamen 14500 junge Tiere und noch 300 Eier.

Der Juli war aber auch deshalb besonders interessant, weil in ihm Männchen auftraten, die an der stark ausgebildeten 1. Antenne (Fig. O a) leicht zu erkennen sind. Namentlich zahlreich waren die Männchen am 3. Juli, wenige waren noch am 29. Juli vorhanden, also nur - ebenso wie in unsern deutschen Seen - eine ganz kurze Zeit. Gleichzeitig mit dem Auftreten der Männchen bildeten die Weibchen Dauereier (Fig. Ob). Die Eier sind rund, haben einen Durchmesser von $0,18 \mathrm{~mm}$ und haben eine feste Schale, die mit kleinen Fortsätzen versehen ist. Ähnliche Eier hat v. DADAY auch gesehen und abgebildet, aber sie für die gewöhnlichen eigentümlich gestalteten Sommereier gehalten. Er schreibt (10): „Sehr interessant sind auch die Eier der Exemplare aus Neu Guinea" von Diaphanosoma sarsi RICH., „indem sich an der Oberfläche ihrer Hülle zahlreiche fingerförmige Fortsätze erheben, wodurch die Eier das Aussehen erhalten, als wären sie dornig“". Die sog. Sommereier sind oval, die große Achse 0,21 mm lang. Diese Dauereier fand ich in nicht sehr großer Zahl, hauptsächlich bildeten die Weibchen noch Sommereier. Das Auftreten der Männchen und Bildung der Dauereier fand im Juli, also kurz vor der größten Trockenheit statt: Der See ist sehr flach, höhere Pflanzen wachsen überall empor, sodaß unter diesen ungünstigen Umständen die Dauereier gebildet werden. Ende Juli bis Anfang September fand ich auch vereinzelt Ephippien, die vielleicht auch zu dieser Art gehören können.

Ceriodaphnia rigaudi RICH. Meine Exemplare entsprechen der C. rigaudi. v. Daday führt von Ceylon (4) C. cornuta G. O. Sars 
an und bildet Übergänge von der $C$. rigaudi zur cornuta ab. Im Colombo-See war diese Art noch nicht gefunden; sie ist daselbst wohl immer zu finden und meist in größerer Zahl. Gleich der erste Fang im Mai lieferte die höchste Zahl, Ende Juni waren nur wenige gefischt, im Juli war die Art wieder häufig in den Fängen, im September wieder selten und im Januar besonders häufig. Ob tatsächlich der Verlauf derartig ist, ist aus den qualitativen Fängen nicht zu sagen; daß Ende Juni die Art aber wirklich selten gewesen ist, glaube ich doch aus der Zahl schließen zu dürfen.

Während der ganzen Zeit fand ich die Sommereier in großer Zahl, von denen jedes Weibchen 2 trägt. Eier und junge Tiere waren so zahlreich, daß nur am 8. Juni und 13. Juli auf eine erwachsene Ceriodaphnia 1 Ei kommt, also im Mittel jedes 2. Weibchen Eier trug. Meist war die Fruchtbarkeit aber größer, am größten am 28. September, wo auf 1 erwachsenes Weibchen 25 Eier resp. junge Tiere kamen. Dieser Fang bildet allerdings das Extrem. Aber auch unter den übrigen Fängen findet sich einer (14. August), in welchem auf 1 erwachsenes Weibchen 6 Eier und Junge kamen. Zwischen $1: 1$ bis 6 Eier: 1 erwachsenes Weibchen schwanken die Zahlen. Ich kann aber nicht sehen, daß eine bestimmte Zeit Regen- oder Trockenzeit - Hauptfortpflanzungszeit ist. Männchen und Dauereier habe ich nicht gefunden.

Moina submucronata Brady. Diese Art hat Brady (5) schon auf Ceylon gefunden, ob im Colombo-See, kann ich nicht angeben. Während der Zeit von Anfang Juni bis Anfang September war diese Daphnide zu finden, also während der Trockenzeit, sie war aber nie so zahlreich wie Ceriodaphnia oder Diaphanosoma, da ich in meinen Fängen höchstens 500 Exemplare fand gegen 14000 resp. 19500 Individuen der andern Arten. Die Hauptzeit scheint von Ende Juni bis Ende Juli zu sein, im August verschwindet sie allmählich, am 8. September fand ich nur 1 Exemplar. Die Fruchtbarkeit war nicht so groß wie bei Ceriodaphnia, wenn man bedenkt, daß ein Weibchen 2-6 Eier trägt. Männchen und Dauereier fand ich ebenfalls nicht bei dieser Art.

Alona punctata v. Daday und

Dunhevedia crassa King. Beide Arten fand ich nur am 28. September in einem Fang der „among weeds“, der also zwischen den den 
See in dieser Zeit erfüllenden höhern Pflanzen (Schilf? oder flutende Pflanzen?) gemacht war. Beide waren in großer Menge vorhanden.

Beide waren von v. DADAY (4) schon für Ceylon nachgewiesen, aber nicht für den Colombo-See, sondern für die Sümpfe am KalawewaSee und Madatugama-See, erstere auch für die Sümpfe am Mahaveliganga-Fluß.

$$
\text { K. Copepoden. }
$$

Cyclops Teuckarti Sars (Fig. P). Von den 7 Cyclopiden, die von v. Daday (4) und Poppe u. Mrázek (11) für andere Gewässer von Ceylon nachgewiesen sind, fand ich nur diese eine Art, die an dem
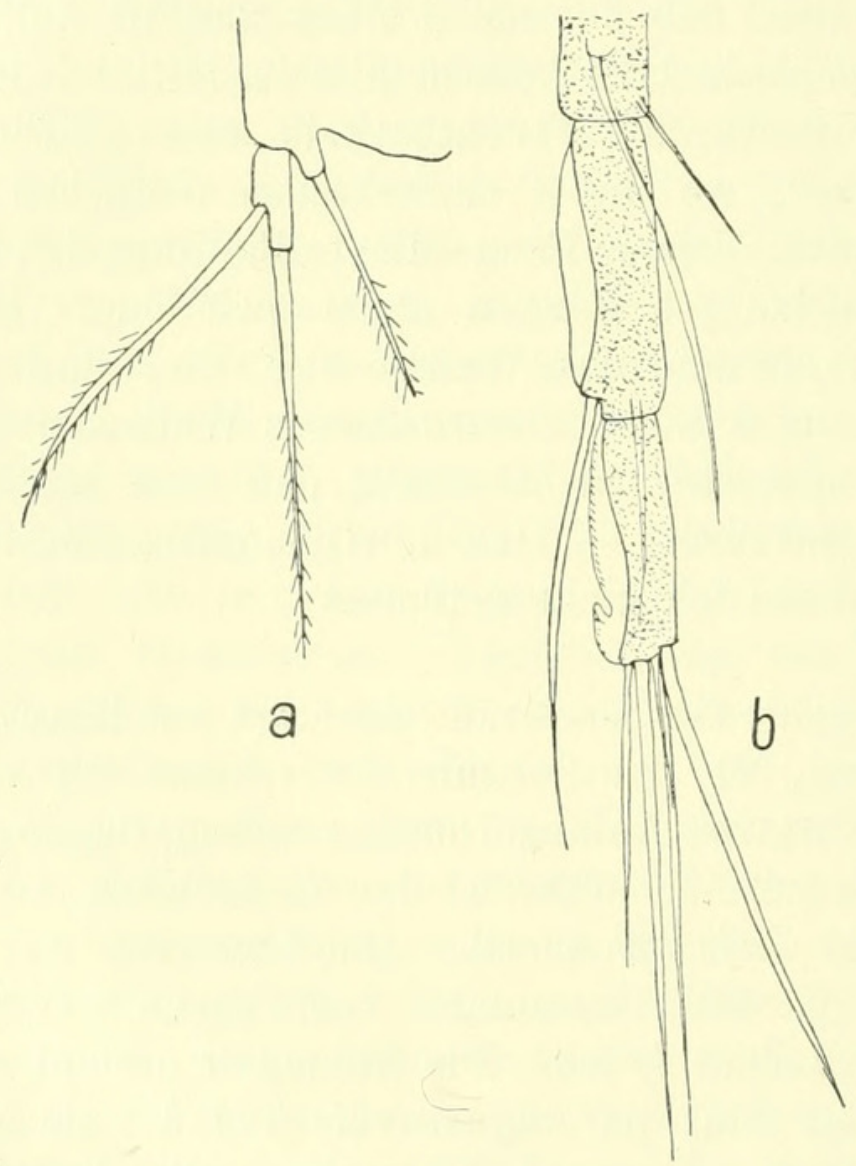

Fig. P.

Cyclops leuckarti.

a 5. Fuß des Weibchens. b die 2 letzten Glieder der 1. Antenne. 300:1.

5. Fuß sowie den Endgliedern der 1. Antenne leicht kenntlich ist (Fig. P). Im Januar und von Mai bis Ende Juli war C. leuckarti häufig, dann fand ich ihn nur noch ganz vereinzelt, ob er weiterhin ganz verschwindet, kann ich nicht angeben. Während derselben Zeit fanden sich auch Männchen. Anfangs waren von allen Er- 
wachsenen $75 \%$ Männchen, dann nahm deren Zahl ab, bis ich am 28. Juni gar keine Männchen fand. Am nächsten Fangtag, 3. Juli, waren deren wieder $25 \%$, und ihre Zahl nahm zu, bis sie am 29. Juli ebenso häufig waren wie die Weibchen. Dann nahm die Zahl der Cyclops so ab, daß nur vereinzelte gefangen wurden. Am 8. September waren fast nur Männchen zu finden, auf 2 Weibchen kamen 20 Männchen. Am 28. September hatten die Weibchen zugenommen, von Männchen erhielt ich aber nur 1 Exemplar, im Januar war die Zahl der Männchen sehr groß. Cyclops trägt 2 Eiersäcke. Am 8. Juni enthielt ein Eiersack im Mittel 9,6 Eier, das ist die kleinste Anzahl, am 29. Juli dagegen 15 Eier als Maximum. Zwischen diesen beiden Extremen schwankte die Eizahl, die geringste Eizahl fällt gerade zusammen mit der höchsten Fangzahl für Cyclops. Überhaupt ist das Eizahlmittel vom Mai bis Mitte Juni geringer als von da bis Ende Juli. Es produziert also Cyclops mit Abnahme der Wassermenge im See verhältnismäßig mehr Eier als während der Regenzeit. Dasselbe zeigt sich auch, wenn wir die Zahl der Eiersäcke mit der der Weibchen vergleichen. Vom 22. Mai bis 15. Juni kommen 2 Eiersäcke auf jedes 3.-7., im Durchschnitt auf jedes 5. Weibchen. Vom 28. Juni bis 29. Juli aber fand ich je 2 Eiersäcke auf jedes 1.-5. Weibchen, im Mittel auf jedes $3^{1} / 2$. Weibchen. Vom August an sah ich keine Eiersäcke mehr. Im Januar kamen ebenfalls auf jedes 5. Weibchen Eiersäcke. Die Zahl der Nauplien war stets grob, ich habe sie leider nicht von denen von Diaptomus unterschieden, vom Mai bis 15. Juni, in welcher Zeit Diaptomus nur ganz spärlich vorkam, werden sie zu Cyclops gehört haben und waren hier entsprechend häufig. Namentlich hoch war die Zahl im Januar, hier handelt es sich um Cyclops, da Diaptomus ganz fehlte. Außer den Nauplien und den erwachsenen Tieren fanden sich häufig die jungen Tiere, d. h. die in den ersten Copepoditstadien, bei denen ich nicht das Geschlecht erkennen konnte. Das Verhältnis dieser jungen Tiere zu den Erwachsenen schwankt sehr, meist waren sie aber häufiger, nur in 3 Fällen waren die Erwachsenen ebenso häufig oder häufiger (15./6., 13.77., 29.7.).

Diaptomus annae n. sp. (Fig. Q). Von Ceylon sind bisher 4 Diaptomus-Arten bekannt geworden. D. orientalis Brady (5), der aber nicht nach der Brady'schen Beschreibung zu erkennen ist, sodaß für ihn die SARs'sche Beschreibung und Abbildungen (12) als Norm gelten müssen, ferner $D$. singalensis von v. DADAY (4), D. lumb- 

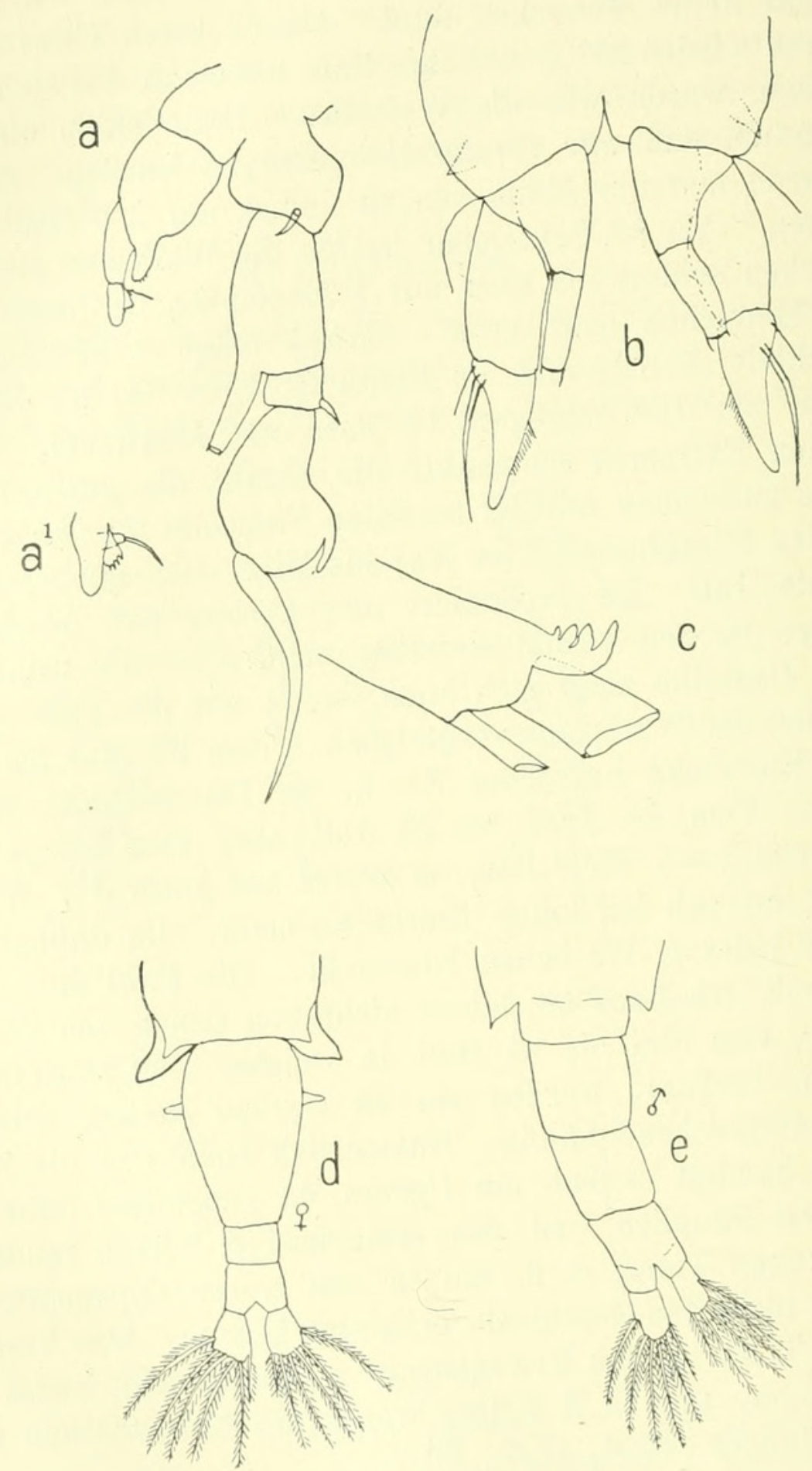

Fig. Q.

Diaptomus annae.

a 5. Bein vom Männchen. $180: 1$. b dasselbe vom Weibchen. 300:1. c Anhang Männchen. $470: 1$ d Hinterende vom Weibchen. am 3. letzten Antennengte Hinterende vom Männchen. $80: 1$. 
holzi Sars von v. Daday (4) und D. drieschi von Poppe u. Mrázer (11). Mit allen 4 Arten und auch mit den übrigen von andern Fundorten bekannt gewordenen Arten stimmen meine Exemplare aus dem Colombo-See nicht überein. Allerdings sind die Unterschiede zwischen all den Arten oft nicht sehr groß, sodaß ich lieber eine Reihe von Arten als Varietäten zu D. orientalis auffassen würde, möchte solch eine Frage aber einem Spezialisten überlassen, da ich nicht genügende Kenntnisse der zahlreichen Arten besitze.

Die von mir gefundenen Diaptomus waren $1,44 \mathrm{~mm} q$ und $1,22 \mathrm{~mm}$ o lang. Der Cephalothorax endet beim $q$ in 2 ziemlich weit ausgezogene Fortsätze, während diese beim o nur ganz gering ausgebildet sind (= lumbholzi, nicht orientalis, drieschi, singalensis).

Abdomen: $q$ 3gliedrig, 1. Segment aufgetrieben, länger als die übrigen zusammen. Genitalsegment mit seitlichem großen Sinnesdorn (= drieschi, bei diesem aber das 2. Segment viel kürzer, und lumbholzi, auch orientalis). of 5gliedrig ohne die Sinnesdornen.

1. Antenne überragt beim $q$ und $\delta$ die Spitze der Furcalborsten (= lumbholzi, bei drieschi etwas kürzer, nur bis zur Furca bei orientalis). 3. letztes Glied beim of mit zierlichem Kamm ohne Lamelle (Fig. Qc) (drieschi kurzer gebogener Fortsatz, lumbholzi spiniform, slightly upturned process, singalensis ohne Stab, hakenförmige Verlängerung oder Hautsaum, orientalis strong somewhat upturned spiniform process).

5. Beinpaar + (Fig. Qb). Außenast 2gliedrig, letztes Glied an der Außenseite mit großer kräftiger und kleinerer Borste, also anders als bei den übrigen 4 in Ceylon gefundenen; ebenso ist der Innenast kräftiger und länger, er erreicht das 2. Giied des Außenasts und trägt am Ende ein paar feine Börstchen.

5. Beinpaar $\delta$, rechter Fuß: 2. Glied mit zylindrischem Anhang, der bis zur Mitte des 4. Glieds reicht (ähnlich lumbholzi). Letztes Glied ungefähr birnförmig, Außendorn klein, dem Glied dicht anliegend, gebogen. Endklaue sichelförmig, von allen 4 andern darin abw.eichend. Endglied des linken Fußes mit gezähnter Lamelle (Fig. Q a und $\mathrm{a}^{1}$ ) und Borste (ähnlich orientalis und drieschi).

Stelle ich noch einmal die unterscheidenden Merkmale der 5 Arten zusammen: 
C. Apstein,

\begin{tabular}{|c|c|c|c|c|c|c|c|c|}
\hline 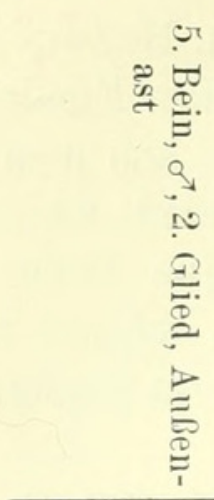 & 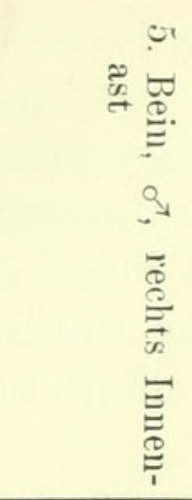 & 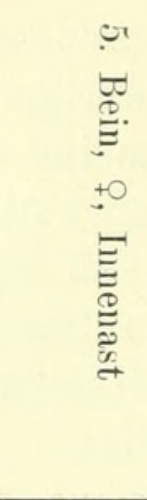 & 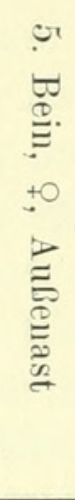 & 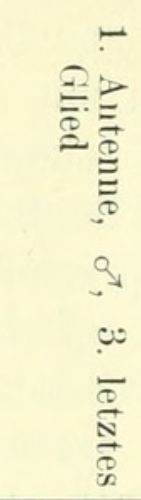 & $\begin{array}{l}- \\
\stackrel{\vec{\oplus}}{\Xi} \\
\stackrel{\Xi}{E}\end{array}$ & 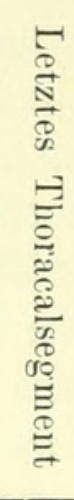 & $\begin{array}{l}\Omega \\
0: \\
D \\
0 \\
\equiv \\
\Xi \\
\vdots \\
\vdots\end{array}$ & \\
\hline 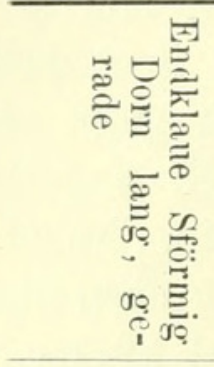 & 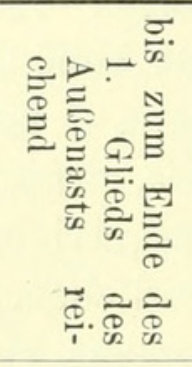 & 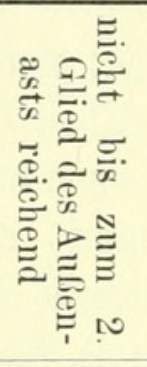 & .ִ. & 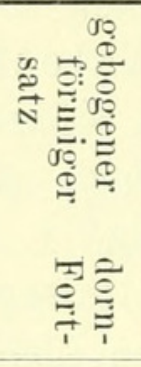 & 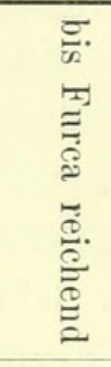 & 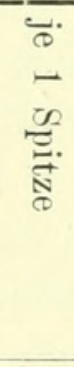 & $\begin{array}{l}\vec{\infty}+0 \\
\vec{c} Q\end{array}$ & 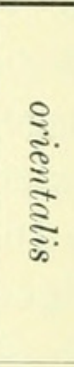 \\
\hline 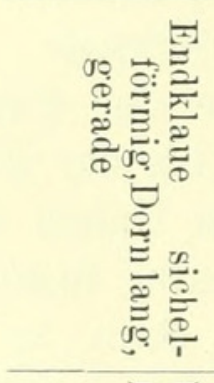 & 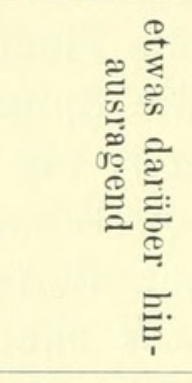 & วิ & 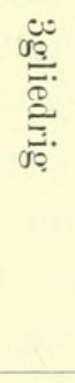 & 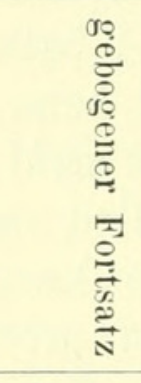 & 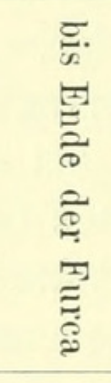 & 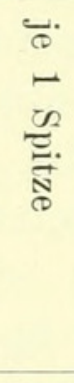 & $\begin{array}{l}50 \\
59\end{array}$ & 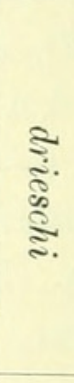 \\
\hline$\widetilde{\circ}$ & 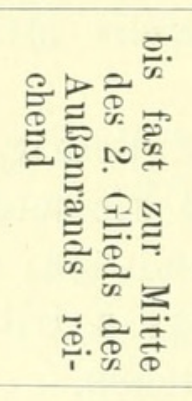 & ?े & & 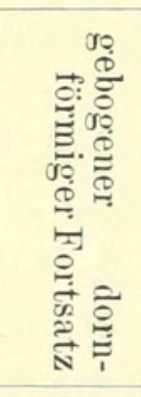 & 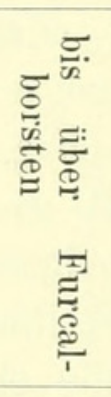 & 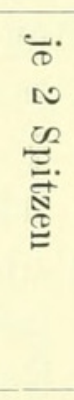 & $\begin{array}{c}\vec{c}+0 \\
1 \\
q\end{array}$ & 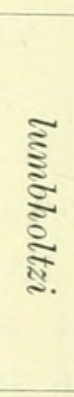 \\
\hline 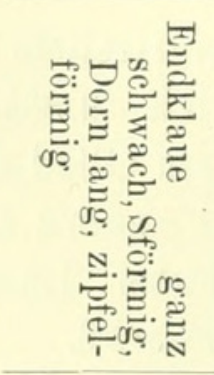 & 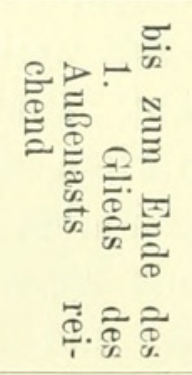 & \% & & 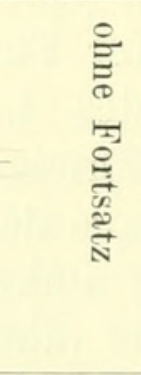 & & 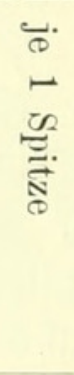 & $\begin{array}{l}-0 \\
0 \\
0\end{array}$ & 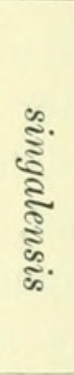 \\
\hline 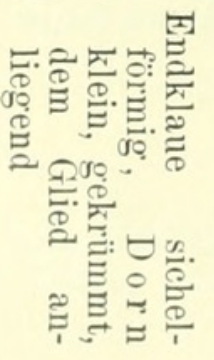 & 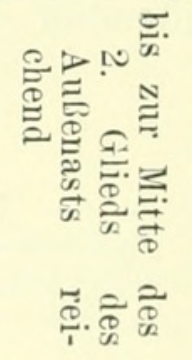 & 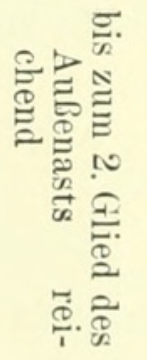 & . & 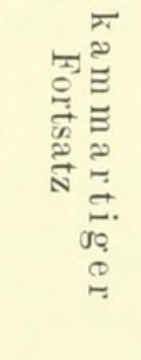 & 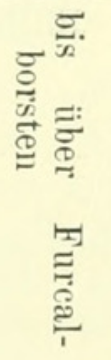 & 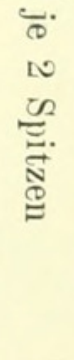 & $\begin{array}{l}\overrightarrow{5}+0 \\
\overrightarrow{5} q\end{array}$ & ฐ్ฐ \\
\hline
\end{tabular}


Nach dieser Zusammenstellung ist der Unterschied von den übrigen klar. In die Tabelle von Giessbrecht u. Schмeil $(13$, p. 67$)$ würde sich Diaptomus so einfügen, wobei ich nur die Nummern des Schlüssels erwähne:

$$
\begin{array}{r}
1-4 \\
4-16 \\
16-40 \\
40-42 \\
42-44 \\
44-45 \\
45-46
\end{array}
$$

Bei 46 würden wir auf $D$. siciloides kommen, von dem sich unsere Art unter anderm durch den kammartigen Fortsatz am 3. letzten Glied der Greifantenne des Männchens unterscheidet.

Diaptomus fehlte im Janıar ganz, im Mai und Anfang Juni war er noch sehr wenig in meinen Fängen enthalten, erst Ende Juni trat er häufig auf und hielt sich bis Ende Juii. Im August scheint die Zahl dann wieder abzunehmen, und Ende September war er nur sehr spärlich. Während der Regenzeit im Mai - ob schon früher? beginnt Diaptomus zu erscheinen, nimmt mit Abnahme des Regens an Zahl zu, wird aber schon zur Zeit der größten Trockenheit 1905 im August - wieder seltner. Er scheint dann nicht wiederzukommen, da er im Januar nach der Regenzeit während des NOMonsuns fehlte. Die Zahlen für die Weibchen scheinen eine regelmäßige Zu- und Abnahme zu zeigen, da es sich aber um qualitative Fänge handelt, so ist nicht anzugeben, ob der Verlauf so regelmäßig ist. Fast stets sind neben den Weibchen auch Männchen gefunden, nur am 8. Juni und natürlich auch am 28. Januar fehlten sie. Das Verhältnis von Männchen zu Weibchen ist schwankend. Am 28. Juni überwogen die Männchen, etwas auch am 27. Mai, sonst waren die Weibchen zahlreicher oder in derselben Zahl wie die Männchen vorhanden. Letzterer Fall trat ein, als die Diaptomus den Höhepunkt der Entwicklung erreicht und überschritten hatten. Meist waren also die Weibchen zahlreicher und zwar im günstigsten Fall 5mal so zahlreich wie die Männchen: 15. Juni. In der Zeit der Abnahme stellt sich das Verhältnis wie $2-3: 1$, d. h. die Männchen waren anfangs seltner, nahmen dann zu, vom August an nahmen sie dann im Verhältnis zu den Weibchen ab.

Diaptomus trägt einen Eiersack. In der Zeit von Mai bis 
Ende Juni kam auf jedes 2.-3. Weibchen ein Eiersack, während der Hauptperiode in der ersten Hälfte des Juli hatte nur jedes 5.-6. Weibchen einen Eiersack, dann besserte sich noch einmal Ende Juli und August das Verhältnis, um am 8. September vor dem Verschwinden der Diaptomus stark zu sinken. 30:1.

Die Zahl der Eier in einem Eiersack war auch verschieden. In der ersten Hälfte des Juni enthielt ein Eiersack 30 Eier, Ende Juni noch 12, also zur Zeit, in der die Weibchen überhaupt viel Eiersäcke trugen. In der übrigen Zeit waren in den Eiersäcken nur 7-9 Eier enthalten, also auch in der Zeit der höchsten Entfaltung wurden nur kleine Eiersäcke produziert, vorher dagegen recht große.

Die Spermatophoren habe ich wohl nicht immer genügend beachtet. Die höchste Zahl habe ich am 28. Juni notiert. Auf jedes Männchen kamen $2^{1 / 2}$ Spermatophoren, während am 13. Juli erst auf jedes 12. Männchen ein Spermatophor zu zählen ist. Produktion an Spermatophoren, Größe des Eiersacks gehen da ganz parallel.

\section{Ostracoden.}

1886 sind von Brady (5) eine Reihe Ostracoden erwähnt resp. neu beschrieben worden, die bei Colombo von HaLy gesammelt waren. Ob der Fundort nun der Colombo-See war, muß dahingestellt bleiben. Später hat v. DadaY (4) eine Reihe von Ostracoden von andern Fundorten Ceylons beschrieben, darunter aber nur eine der BRADYschen Arten erwähnt. Ich habe bei dem mir vorliegenden Material mein Augenmerk besonders auf die Arten von BRADY gerichtet, wobei ich hervorheben muß, daß eine Identifikation nicht leicht und nicht immer sicher ist, da BRady nur Zeichnungen der Schale gibt. Ich gebe Abbildungen der wichtigsten Extremitäten, sodaß eine Erkennung der Arten jetzt leichter gelingen wird. Lange Beschreibungen kann ich mir bei den Arten sparen, da die Figuren für sich selbst sprechen und noch besser als viele Worte.

Cypris purpurascens Brady (Fig. R). Bei weitem war diese Art im Colombo-See am häufigsten und vielleicht die einzige, die pelagisch lebt. Auch Brady bezeichnet sie als die häufigste Art. Die Schale trägt die von Brady (5) erwähnten Purpurflecke. Die Länge der Schale beträgt beim Weibchen 0,50 mm, während Bвadr $1 \mathrm{~mm}$ erwähnt, das Männchen ist etwas kleiner, $0,45 \mathrm{~mm}$ lang. Schale am Vorder-, Unter- und Hinterrand beborstet (R a u. d). 

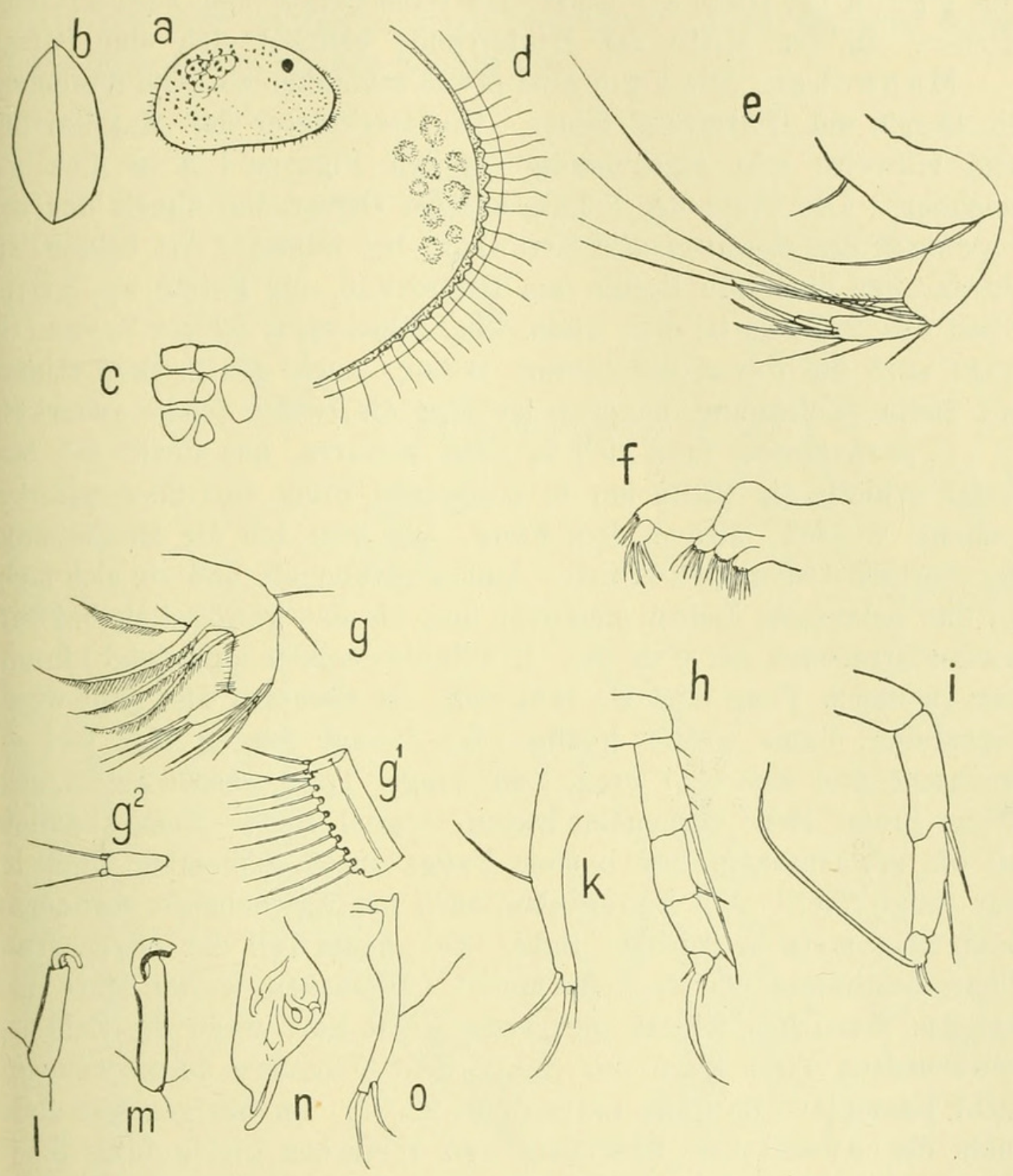

Fig. R.

Cypris purpurascens. a-k $+, 1-0 \quad \sigma^{\top} . \quad c-0 \quad 180: 1$.

a Schale von der Seite, b vom Rücken. 33:1. c Muskelansatz. 180:1. d Vordercand der rechten Schale. e 2. Antenne. f Kauteil der 1. Maxille. g Taster der Vandibel. $\mathrm{g}^{1}$ Borsten des 1 . Glieds stärker vergrößert. $\mathrm{g}^{2}$ Durchschnitt durch die Borste. h 1 . Bein, i 2 . Bein, k Furca. $1-0 \sigma^{7}$. l, m Greiftaster des Maxillarfußes. n Penis. o Furca.

Der Taster der Mandibel trägt an dem 1. Glied (Fig. Rg) t Borsten, die im Querschnitt oval sind. An einer schmalen Seite tehen auf kleinen Höckern zwei Reihen von kleinen Fiedern ( $\mathrm{g} 2$ ). Jas letzte Glied des 2. Beins trägt eine kräftige, lange Borste (i). 
Die Furca (k) trägt am Ende eine feine Borste und zwei kräftige Klauen. In der Mitte des Hinterrands befindet sich eine Borste.

Männchen. Die Furca ist etwas schlanker als beim Weibchen, die Borste am Hinterrand feiner. Die Greiftaster der Maxillarfüße sind teilweise sehr starkwandig (in den Figuren 1 u. m dick gezeichnet). Die Form der Schale unseres Ostracoden ähnelt der von Cyprinotus dentato-marginatus SARS (14). Bei letzterer Art besitzt die Furca aber nicht die Borste am Hinterrand, die Borste am letzten Glied des 2. Beins ist nur klein. Bei Physocypris bullata Vavra (15) trägt auch die Furca die hintere Borste, auch das 2. Bein stimmt mit meiner Zeichnung, dagegen ist aber die rechte Schale gebuckelt

C. purpurascens fand sich in allen Monaten, aus denen ich Material erhielt, sie fehlte nur in dem einen Fang vom 28. September „among weeds", also in dem Fang, aus dem ich die Hauptmenge der übrigen Ostracoden erhielt. Daher glaube ich, daß sie sich mehr an das pelagische Leben angepaßt hat, als das gewöhnlich bei Süßwasserostracoden der Fall ist. Die Zahlen gehen auffallend hin und her, in einem Fang sind sie zahlreich, im nächsten nur sehr wenig vorhanden, dann wieder häufig. Im Januar fanden sich viel erwachsene und eine sehr große Zahl junger Tiere, ebenso am 29. Juli. Wenn junge Tiere vorhanden waren, waren sie 7-20mal häufiger als die erwachsenen, nur in dem Fange am 8. September fand ich nur junge Tiere. Oft waren aber auch nur Erwachsene vorhanden, dann aber stets in geringer Zahl. Bei einem Teil der erwachsenen Tiere bestimmte ich das Geschlecht. Weibchen waren stets vorhanden, Männchen fehlten mehrmals, nicht nur, wenn die Zahl der erwachsenen Tiere klein war (22./5., 28.9.), sondern auch, wenn ich mehr Exemplare im Fang hatte $(8 . / 6,29.7$.). Im übrigen war mehrmals die Anzahl der Männchen und Weibchen gleich (Juli 3, 13, August 14), in andern Fällen überwog die Zahl der Weibchen, so Juni 15 s $: q=1: 2$, und $\delta: q=1: 5-6^{1} / 2$ am 29. Mai und 28. Juni. Ein gesetzmäßiges Verhalten konnte ich nicht erkennen.

Stenocypris major BaIRD (= St. malmcolmsoni BRAdy) (Fig. S). Dieser prächtige Ostracode war nur einmal in einem Planctonfang vom 22. Mai in einem Exemplar vorhanden, dagegen fand ich mehrere Exemplare in dem Fang vom 14. August, der Bodenmaterial enthielt, und dann in dem Fang vom 28. September „among weeds".

Die Furca sowie die an ihr befindlichen Klanen sind an der Hinterseite mit kräftigen Zähnen besetzt, die so dicht stehen, daß 
das Bild einer Säge zustande kommt. Einige Autoren (5) zeichnen statt der sehr kräftigen stark chitinigen Zähne einfache Borsten oder doch solche an den Klauen (14).

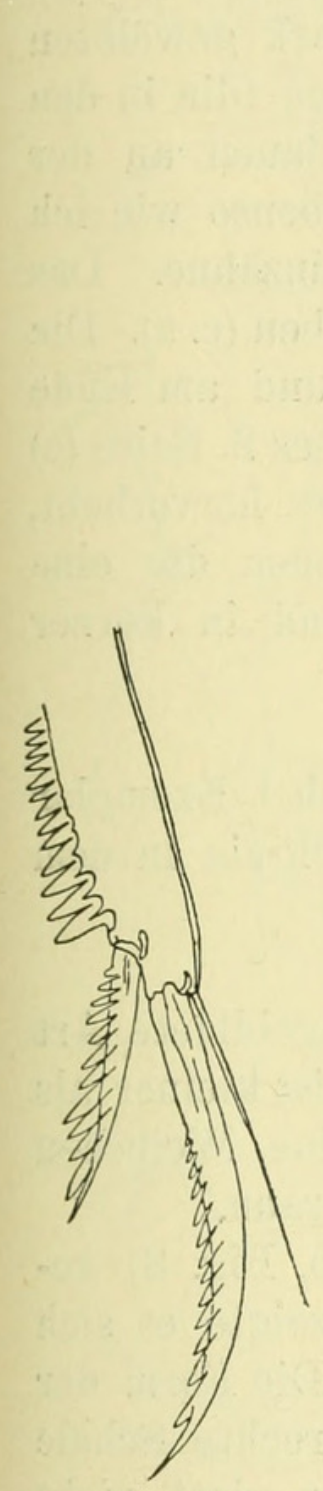

Fig. S.
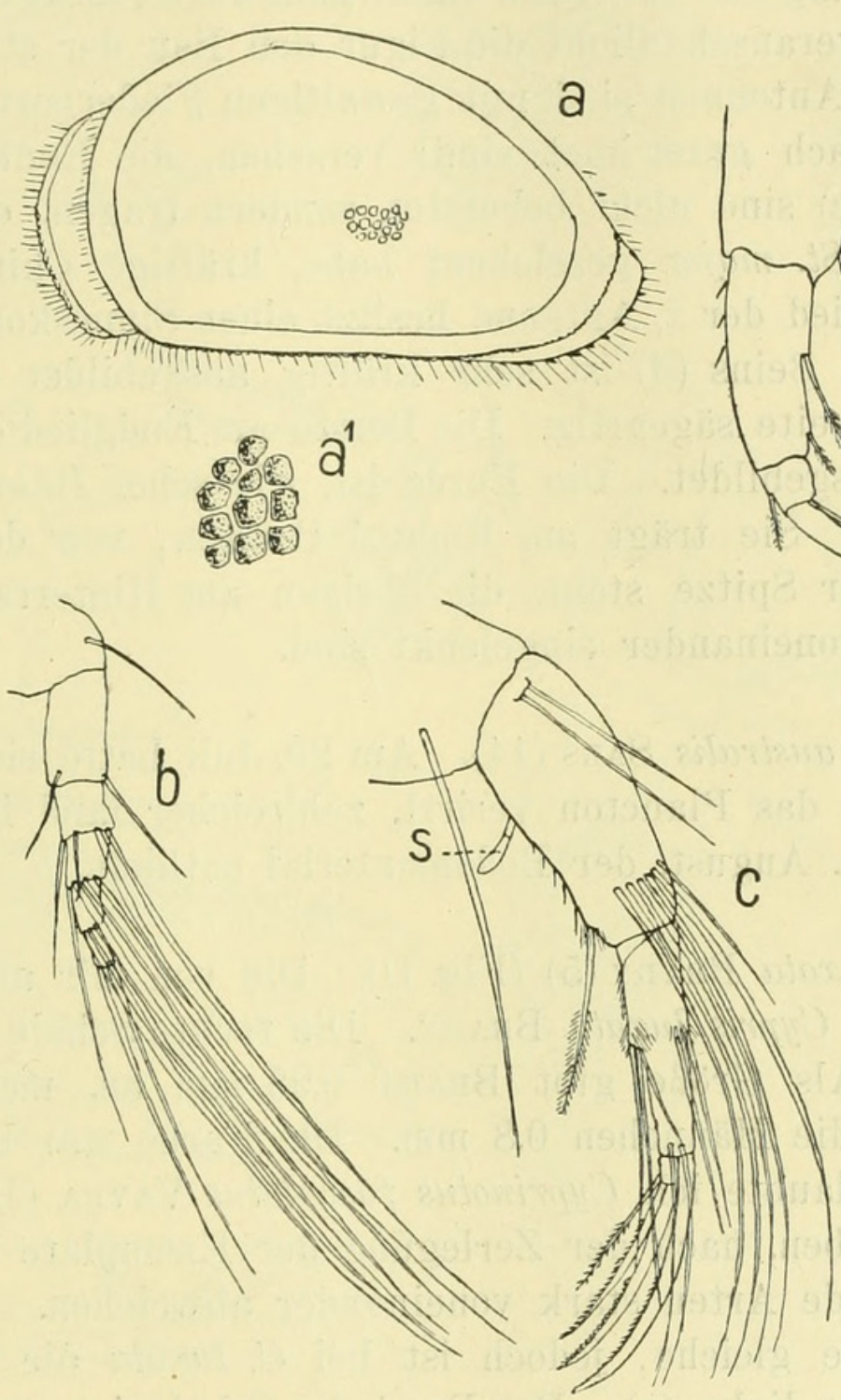

Fig. T.

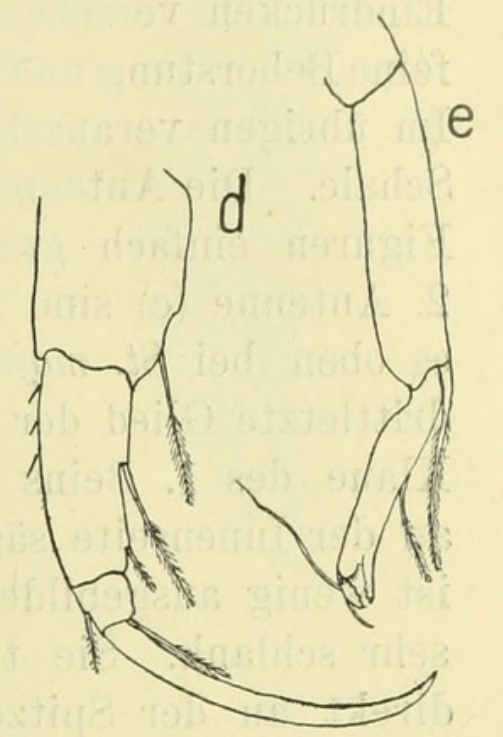

Fig. S. Stenocypris major. Furca. 180:1.

Fig. T. Chlamydotheca subglobosa. a Rechte Schale von innen. 33:1. $a^{1}$ Struktur der Schale. 100:1. b 1. Antenne. c 2. Antenne. d 1. Bein. e 2. Bein. f Furca. b-f $100: 1$.

Chlamydotheca subglobosa Sowerby (Fig. T). BRADy (5) erwähnt diesen Ostracoden schon von Ceylon und gibt von ihm Abbildungen der Schale (5, Fig. 24-27), nach denen die Art gut wiederzuerkennen ist. Ferner erwähnt er, daß die Furca sehr dünn ist. Diese Merkmale stimmen gut für eine Reihe von Ostracoden, die ich in dem

Zool. Jahrb. XXV. Abt. f. Syst. 
Fang vom 28. September „among weeds“ erhielt. In andern Fängen war kein Exemplar enthalten.

Die sehr feste Schale ist an der ganzen Oberfläche mit tiefen Eindrücken versehen $\left(\mathrm{a}^{1}\right)$, trägt vorn, an der Unterseite und hinten feine Beborstung und außerdem hinten ganz kurze, dicke Stachelchen (a). Im übrigen veranschaulicht die Figur den Bau der stark gewölbten Schale. Die Antennen sind mit gewaltigen Fiederborten (die in den Figuren einfach gezeichnet sind) versehen, die Endklauen an der 2. Antenne (c) sind nicht beborstet, sondern tragen, ebenso wie ich es oben bei St. major gezeichnet habe, kräftige Chitinzähne. Das drittletzte Glied der 2. Antenne besitzt einen Sinneskolben (c. s). Die Klaue des 1. Beins (d) ist sehr kräftig ausgebildet und am Ende an der Innenseite sägeartig. Die Borste am Endglied des 2. Beins (e) ist wenig ausgebildet. Die Furca ist, wie schon Brady hervorhebt, sehr schlank. Sie trägt am Ende 4 Borsten, von denen die eine direkt an der Spitze steht, die übrigen am Hinterrand in kurzer Entfernung voneinander eingelenkt sind.

Ilycypris australis SARS (14). Am 29. Juli hatte sich 1 Exemplar dieser Art in das Plancton verirrt, zahlreicher fand ich sie in dem Fang vom 14. August, der Bodenmaterial enthielt.

Cypris luxata Brady (5) (Fig. U). Die von mir abgebildete Art halte ich für Cypris luxata BRAdy. Die rechte Schale ist kleiner als die linke. Als Größe gibt Brady $0,98 \mathrm{~mm}$ an, meine Weibchen maßen 0,97, die Männchen $0.8 \mathrm{~mm}$. Die Farbe war braun.

Zuerst glaubte ich Cyprinotus fasculatus Vavra (15, Fig. 8) gefunden zu haben, nach der Zerlegung der Exemplare zeigte es sich aber, daß beide Arten stark voneinander abweichen. Die Form der Schale ist die gleiche, jedoch ist bei $C$. luxata die rechte Schale kleiner als die linke (a). Der Rand der Schale ist vorn glatt, nicht krenuliert, wie bei $C$. fasculatus. Die Furca trägt noch über der Mitte eine Borste, die bei C.fasculatus weiter nach dem Ende steht. Dann sind auch die Greiftaster beim Männchen ganz anders gebaut.

Das 2. Bein trägt keine Borste am Endglied. Die weitern anatomischen Merkmale zeigen die Figuren.

Die Art fand sich in mehreren Exemplaren am 14. August und 27. September im Bodenmaterial und „among weeds“, häufiger in ersterm.

Von den 10 Exemplaren an ersterm Fundort waren 6 Männchen 

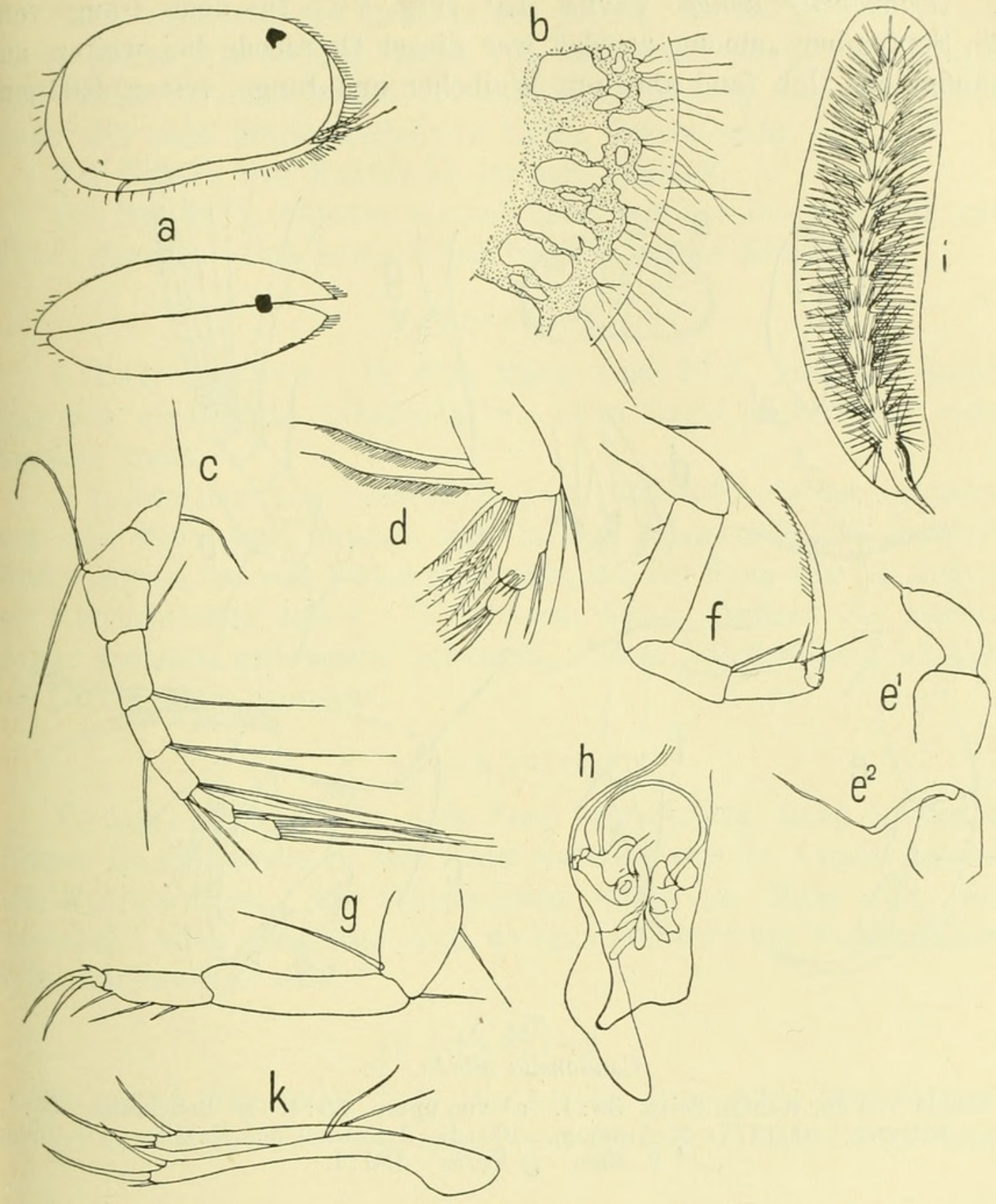

Fig. U.

Cypris luxata.

a Schale des Weibchens. 33:1. b Vorderrand von innen. 180:1. c-k Männchen. $180: 1$. c 1 . Antenne. d Taster der Mandibel. $e^{1}, e^{2}$ Greiftaster der Maxillarfüße. f 1 . Bein. g 2. Bein. h Penis. i Ejakulationsorgan. k Furca.

und 4 Weibchen, an letzterm 2 Männchen und 1 Weibchen. Also in beiden Fällen die Männchen häufiger als die Weibchen. 
Candonella albida Vavra (15) (Fig. V). In dem Fang vom 28. September „among weeds“ war dieser Ostracode bei weitem am häufigsten. Ich fand aber nur Weibchen und Junge, erstere $0,64 \mathrm{~mm}$
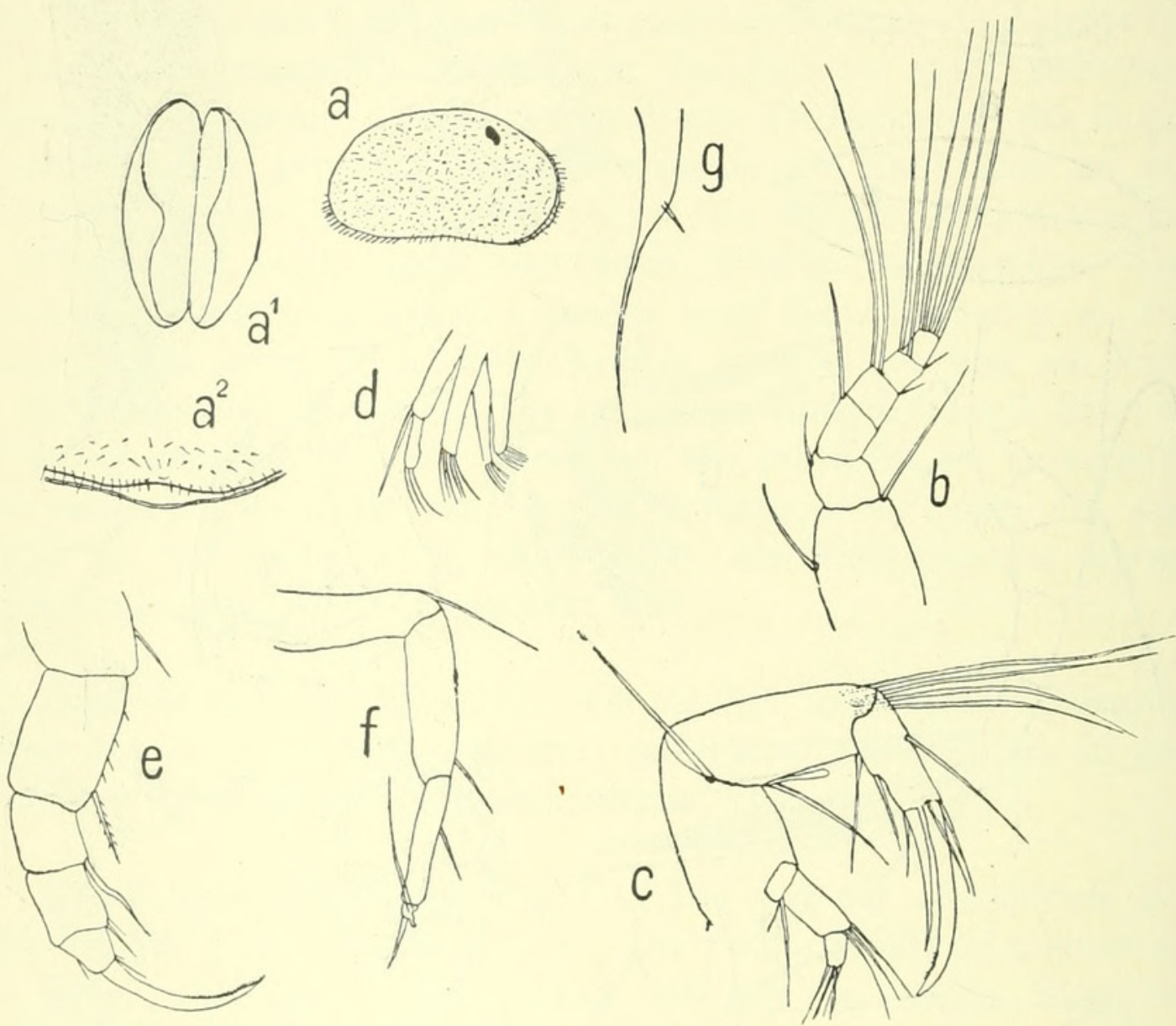

Fig. V.

Candonella albida. ㅇ.

a Schale von der rechten Seite. 33:1. a ${ }^{1}$ von unten 33:1. $a^{2}$ Bauchrand. 33:1. b 1. Antenne. 100:1. c 2. Antenne. 100:1. d Kauteil der Maxille. e 1. Bein. f 2. Bein. g Furca. $180: 1$.

lang, was mit der Angabe von Vavra (15) stimmt. C. albida ist im Wembere-Sumpf bei Tabora gefunden, meine Ceylon-Exemplare stimmen mit erstern überein, einige kleine Abweichungen fand ich jedoch.

Die Schalenform ist übereinstimmend, daß aber die Borsten alle nach hinten gerichtet sind, wie VAVRa schreibt, war bei meinen Exemplaren nicht der Fall, möglich, daß durch den Druck des Deckglases die Regelmäßigkeit der Lagerung gestört war. Sieht man die Schale von der Bauchseite $\left(a^{1},^{2}\right)$, so fällt die Vorbuchtung in der Mitte des Schalenrands auf. 
Ganz eigenartig ist der Bau der Furca, die bei meinen Exemplaren genau wie die bei denen von Ost-Afrika ist $(\mathrm{g})$ : Die Furca geht direkt in einen langen borstenartigen Anhang über, an dessen Basis eine nach hinten gerichtete kleine Borste steht.

Der Kauteil der Maxille (d) ist sehr schlank.

Der Bau der 2. Antenne stimmt nicht mit der Beschreibung von Vavra überein. Das letzte Glied trägt 2 starke Klauen.

\section{In secten.}

Libellenlarve. In dem Fang vom 28./9. „among weeds" fand sich eine große Libellenlarve, deren Zugehörigkeit ich nicht angeben kann.

M ückenlarven. Am 29.7., 14./8. und 8.9., als der ColomboSee sehr flach war, fanden sich in den Planctonfängen mehrere Mückenlarven, die vom Boden stammten, wie der Fang vom 14. August mit Bodenmaterial zeigte. In letzterm Fang steckten die Larven in aus Schlamm gefertigten Gehäusen. Etwas Näheres kann ich über die Larven nicht angeben.

N. Arachnoideen.

Hydrachniden. In dem Fang vom Januar, dann in den 3 Fängen im Juli sowie in dem Bodenmaterial vom 14. August fanden sich Hydrachniden. Am zahlreichsten waren sie Mitte Juli. Ich beschränke mich auf diese Notiz, da Herr Könıcke die Hydrachniden näher untersuchen wird.

\section{O. Bryozoen.}

In einem Exemplar fand ich am 28. Juni einen Statoblasten, der genau die gleiche Form zeigte wie der von Plumatella princeps.

\section{Teil.}

Zusammensetzung des Planctons in den verschiedenen Monaten.

Aus der vorangehenden Zusammenstellung ging schon hervor, daß das Plancton in den verschiedenen Monaten ungleich zusammengesetzt ist, da die meisten Organismen ein periodisches Auftreten haben.

Der J an u ar ist der trockenste Monat während des NO-Monsuns, da in ihm im Mittel nur $47 \mathrm{~mm}$ Regen fallen. In ihm setzte sich 
das Plancton namentlich aus tierischen Vertretern zusammen, während wir von Pflanzen in großer Zahl nur Clathrocystis finden (siehe Tabelle im Anhang).

Unter den Tieren waren wie gewöhnlich vertreten Rädertiere, Daphniden, Copepoden, Ostracoden und Hydrachniden.

Unter den Rädertieren, von denen 7 Arten vorkamen, war am häufigsten Pedalion mirum, die Zahl war fast doppelt so hoch wie die aller andern Rädertiere zusammen. Nächstdem fand sich viel Brachionus forficula var. levis, Br. amphiceras var. borgerti, Anuraea valga var. tropica, Triarthra longiseta und Asplachna brightwelli. Die meisten Rädertiere waren in reger Fortpflanzungstätigkeit begriffen, die Zahl der Eier zu den Weibchen schwankt von $1: 10$ bis $1: 1$. Nur von Pedalion und Triarthra habe ich keine Eier notiert.

Unter den Daphniden vermißte ich Moina submucronata, die erst im Juni zu finden war. Diaphanosoma und Ceriodaphnia waren sehr zahlreich, ebenso deren Eier.

Von Copepoden fand sich nur Cyclops leuckarti. Namentlich häufig war die Larve dieses Copepoden, 54 mal so viel Nauplien wie Weibchen. Aus andern Monaten kann ich das Verhältnis nicht angeben, da dann die Larven zweier Copepoden gemischt vorkommen und ich nicht beide getrennt habe.

Der stets pelagisch vorkommende Ostracode Cypris purpurascens war auch in zahlreichen, meist jungen Exemplaren vertreten. Schließlich erwähne ich einige Hydrachniden, wodurch die Aufzählung der vorhandenen Organismen erschöpft ist.

Februar bis April. Diese Monate liegen zwischen dem NOund SW-Monsun, die Regenmenge steigt vom Januar an und erreichte im April schon $159 \mathrm{~mm}$ (siehe Fig. B). Während dieser Zeit ist mir das Verhalten des Planctons, da ich keine Fänge aus diesen Monaten habe, unbekannt. Aus der Kombination von Januar und Mai scheint aber Folgendes hervorzugehen. Die Pflanzen, vertreten durch Clathrocystis, herrschen allein vor.

Asplanchna, die ein sehr unregelmäßiges Auftreten zeigt, schien verschwunden zu sein. Diaphanosoma war ebenfalls aus dem Plancton geschieden oder war vielleicht nur sehr selten geworden. Im übrigen läßt sich kaum eine Vermutung über diese Monate aussprechen.

M ai. Die Regenzeit hat ihren Höhepunkt erreicht, 1905 mit $344 \mathrm{~mm}$. Noch immer sehen wir Clathrocystis als Hauptvertreter der Pflanzen. Von Protozoen war vorübergehend Coleps hirtus häufig. Die Rädertiere haben sich gegen den Januar nicht sehr geändert, 
nur Asplanchna fand sich vereinzelt, Triarthra war seltner, ebenso Anuraea valga var. tropica, neu aufgetreten waren eine nicht näher bestimmte Synchaeta, eine Mastigocerca und Brachionus falcatus var. $\beta$. Diaphanosoma findet sich sehr spärlich. Die Copepoden zeigen eine Änderung, indem zu Cyclops jetzt auch Diaptomus, allerdings nur in wenigen Exemplaren, hinzugekommen ist.

Juni. Die Regenhöhe hat sehr abgenommen, sie beträgt nur $113 \mathrm{~mm}$, also ${ }^{1 / 3}$ von der im Mai. Das Bild ändert sich jetzt. Während bisher unter den Pflanzen allein Clathrocystis vorherrschte, wird sie jetzt von Melosira verdrängt, die gegen das Ende des Monats im Fang ganz überwog. Andere Algen, wie Closterium, kleine Volvocineen, Pediastrum fanden sich in verschiedener Häufigkeit, spielten aber gegenüber Melosira keine Rolle. Synchaeta fand sich Anfang des Monats häufig, nahm dann aber an Zahl ab, ebenso Brachionus falcatus var. $\beta$, der schon im Mai vereinzelt aufgetreten war. Fast nur auf diesen Monat beschränkt waren Polyarthra und Brachionus pala var. willeyi. Häufig fanden sich im Plancton, namentlich gegen Ende des Monats, Triarthra longiseta, Anuraea valga var. tropica, Brachionus rubens, Pedalion mirum. Brachionus amphiceras var. borgerti war Mitte Monat häufiger und wurde viel von dem oben in Fig. H abgebildeten Parasiten geplagt. Diaphanosoma war zahlreich, dagegen schien Ceriodaphnia abgenommen zu haben, und Moina war in diesem Monat neu aufgetreten.

Cyclops blieb häufig und Diaptomus nahm gegen Ende des Monats stark zu. Ersterer war oft besetzt mit Colacium und Vorticellen.

Juli. In diesem Monat, der im Durchschnitt der trockenste des ganzen Jahrs ist, waren im Jahr 1905 noch $32 \mathrm{~mm}$ Regen gefallen. Der See wird daher wohl weniger Wasser führen, also für manche Organismen ungünstigere Verhältnisse zeigen, für andere gerade umgekehrt. So findet sich in diesem Monat die größte Mannigfaltigkeit der Algen. Melosira dominierte immer noch, aber Clathrocystis fällt schon mehr in die Augen. Häufiger war auch Pediastrum duplex var. reticulatum, während die übrigen Arten zum Teil nur an einem Fangtag in größerer Zahl auftraten, um dann spärlich zu werden oder zu verschwinden. Von Protozoen fand sich Coleps auch noch in diesem Monat in nicht sehr großer Zahl. Die Rädertiere verhalten sich verschieden. Häufig fanden sich noch Anuraea valga var. tropica, Triarthra longiseta, Brachionus amphiceras var. borgerti, andere waren verschwunden oder verschwanden wie 
Polyarthra platyptera, Brachionus pala var. willeyi, Synchaeta, Brachionus caudatus, Brachionus rubens, Pedation mirum. Neu traten auf oder wieder auf Notops macrurus, Brachionus falcatus var. $\beta$, während Asplanchna sehr wechselte.

Bei den Daphniden fand ich interessante Verhältnisse, indem ich für Diaphanosoma Männchen und Dauereier feststellen konnte, alle Arten aber noch häufig fand.

Neu traten Hydrachniden auf und am Ende des Monats Mückenlarven. Letztere mögen im Wasser getrieben haben, ich fand sie im August am Boden in Schlammröhren, im Juli ohne diese.

A ugust.' Der regenärmste Monat des Jahrs 1905, da nur $15 \mathrm{~mm}$ Regen gefallen waren. Von Algen überwog wieder Clathrocystis; Melosira war nicht mehr so häufig, häufiger trat aber Closterium lunula var. coloratum auf.

Das Protozoon Coleps war verschwunden. Rädertiere waren wie im Vormonat, die Daphniden hatten stark abgenommen, ebenso die Copepoden.

September. Die Regenzeit setzte in diesem Monat wieder ein, $273 \mathrm{~mm}$ wurden gemessen. Melosira verschwindet aus dem Plancton, sodaß wir hauptsächlich Clathrocystis finden.

Die Rädertiere verschwinden zum Teil wie Notops macrurus und Brachionus falcatus var. $\beta$, andere, die verschwunden waren, treten noch einmal in geringer Zahl auf, wie Polyarthra, Brachionus caudatus, Brachionus pala var. willeyi, um aber Ende Monat schon wieder zu fehlen. Neu tritt wieder auf Brachionus forficula var. levis. Daphniden und Copepoden sind ganz spärlich geworden, produzieren auch nur wenig Eier.

Wie sich die Organismen weiter während der großen Regenzeit verhalten, läßt sich wegen des großen Zwischenraums zwischen September bis Januar auch nicht annähernd sagen.

Die Trockenzeit Juli, August, auch Juni können wir wohl dazu rechnen, zeigt uns ihren Einfluß auf das Plancton einmal wohl durch den geringern Wassergehalt des Sees und dann, daß weniger Nährstoffe dem See zugeführt werden.

Im Juli ist noch üppiges Leben im See, im August ändert sich das, Daphniden und Copepoden werden spärlich, eine Art der erstern hat sich durch Dauereibildung auf die ungünstigen Verhältnisse vorbereitet. Von Rädertieren verschwinden manche schon im Juli, die vorher noch recht häufig gewesen waren. Andrerseits treten im Juli die Algen mannigfaltiger auf, und auch unter den Rädertieren gibt 
es einige, die jetzt erst erscheinen oder doch nach kurzer Pause wiederkommen. Die ungünstigen Verhältnisse finden sich bis in den September, in welchem Monat die größere Regenmenge doch schon Abhilfe schaffen mußte.

\section{Teil.}

\section{Fauna des Colombo-Sees.}

Aus dem Colombo-See sind bisher nur von Brady (5) und von v. Daday (4) Tiere bekannt geworden. Ersterer erhielt sein Material aus der Nähe Colombos, ob aus dem Colombo-See, weiß ich nicht, will es aber für die Zusammenstellung annehmen, letzterer hat sowohl Material aus dem Colombo-See als auch aus andern Gewässern Ceylons untersucht. Bei der Aufzählung bedeutet hinter dem Artnamen $\mathrm{BR} .=$ Brady, Dad. $=$ v. Daday, A. = Apstein. Wegen der Algen verweise ich auf meine vorläufigen Bemerkungen oben, dann aber auf Lemmermann, dessen Arbeit aber noch nicht erschienen ist.

Protozoen: Arcella vulgaris EHBG.

Coleps hirtus EHBG.

(Ceratium hirundinella ${ }^{1}$ ) O. F. M.

Cölenteraten: Hydra sp.

Rotatorien: Rotifer vulgaris EHBG.

Asplanchna brightwelli

Synchaeta $s p$.

Mastigocerca $\mathrm{sp}$.

Notops macrurus

Triarthra longiseta

Brachionus caudatus BARR. et DAD.

Anuraea valga var. tropica APsT.

Brachionus rubens EHBG.

Brachionus amphiceras var. borgerti APsT.

Brachionus pala var. willeyi Apst.

Brachionus forficula var. levis APST.

Brachionus falcatus var. $\beta$ WEBER

Polyarthra platyptera EHвG.

Pedalion mirum HuDs.

Branchiopoden:

Daphniden :
Cyclestheria hislopi BRADY

Diaphanosoma singalense v. DAD.

Ceriodaphnia rigaudi $\mathrm{R} I \mathrm{CH}$.
DAD.

A.

DAD.) .

A.

DAD.

A.

A.

A.

A.

A.

A.

A.

A.

A.

A.

A.

A.

A.

A.

BR. A.

A.

A.

1) Ich rechne Ceratium zu den Pflanzen, da v. DadaY es aber unter Protozoen aufführt, habe ich es hier auch aufgenommen, aber eingeklammert. 
Moina submucronata BRADY

Guernella ceylonica v. DAD.

Macrothrix triserralis BR.

Iliocryptus halyi $\mathrm{BR}$.

Alonopsis orientalis $\mathrm{v}$. DAD.

Alona punctata v. DAD.

Alona macronyx v. DAD.

Alona longirostris v. DAD.

Alona acanthocercoides FischeR

Alonella karua (KING)

Dunhevedia crassa (KING)

Dunhevedia serrata v. DAD.

Chydorus sphaericus $v$. parvus $\mathrm{v}$. $\mathrm{D}_{\mathrm{AD}}$.

Chydorus sphaericus $v$. ceylanicus v. DAD.

Chydorus barroisi (RICH.)

Copepoden: Diaptomus orientalis $\mathrm{BR}$.

Diaptomus annae APst.

Cyclops leuckarti Claus

Ostracoden:

Insecten :

Cypridopsis marmorata BRADY

Cypridopsis globosa BRADY

Stenocypris major BAIRD

Iliocryptus australis G. O. SARS

Cyprinotus cingalensis BRADY

Cypris monilifera BRADY

Cypris luxata BRADY

Cypris purpurascens BRADY

Cypris halyi BRADY

Cypris tenuicauda BRADY

Cypris furfuracea BRADY

Chlamydotheca subglobosa $\mathrm{SAW}$.

Candonella albida VAVRA

Libellenlarve

Mückenlarve

Hydrachniden: Atax nodosus v. DADAY

2 Arten

Bryozoen: Statoblast v. Plumatella
BR. A.

DAD.

BR.

Br.

DAD.

A.

DAD

DAD.

DAD.

DAD.

A.

DAD.

DAD.

DAD.

DAD.

BR.

A.

A.

BR.

BR.

A.

A.

BR.

BR.

BR. A.

BR. A.

BR.

BR.

BR.

BR. A.

A.

A.

A.

DAD.

A.

A. 


\begin{tabular}{lrl} 
Das sind Protozoen & 2 & Arten \\
Cölenteraten & 1 & \\
Rotatorien & 15 & Arten resp. Varietäten \\
Branchiopoden & 1 & $"$ \\
Daphniden & 17 & $"$ \\
Copepoden & 3 & $"$ \\
Ostracoden & 13 & $"$ \\
Insecten & 2 & $"$ \\
Hydrachniden & 2 & $"$ \\
Bryozoen & 1 & \\
\cline { 2 - 3 } & 57 Arten
\end{tabular}

von Tieren, davon 29 neu für den Colombo-See

Von Ceylon sind namentlich von v. DadAy eine große Anzahl Arten aufgeführt: rechne ich die oben aufgezählten neu gefundenen hinzu, so sind von Ceylon bekannt:

\begin{tabular}{lcc} 
& bisher & jetzt \\
Protozoen & $\left.28^{1}\right)$ & 29 \\
Cölenteraten & 0 & 1 \\
Rotatorien & 46 & 59 \\
Oligochäten & 3 & 3 \\
Turbellarien & 2 & 2 \\
Gastrotrichen & 2 & 2 \\
Branchiopoden & 1 & 1 \\
Daphniden & 36 & 36 \\
Copepoden & 12 & 13 \\
Ostracoden & 18 & 19 \\
Insectenlarven & 0 & 2 \\
Hydrachniden & 14 & 14 ? \\
Bryozoen & 0 & 1 \\
\cline { 2 - 2 } & & 182 Arten von Ceylon.
\end{tabular}

1) Ich rechne nicht mit: Ceratium hirundinella, Peridinium tabulatum, Volvox aureus. 


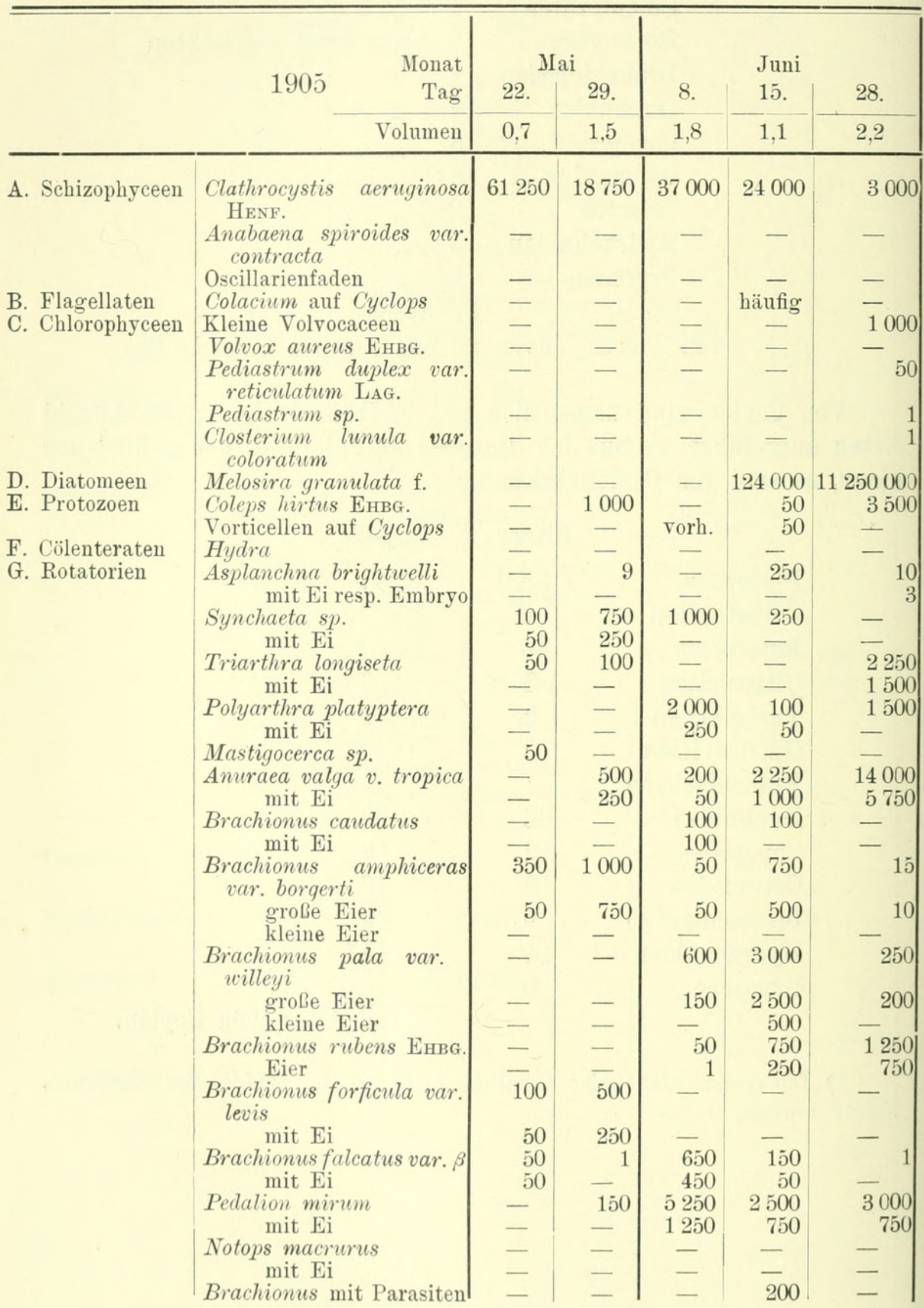




\begin{tabular}{|c|c|c|c|c|c|c|c|c|}
\hline & & & & & & & & \\
\hline & Juli & & August & Sep & mber & August & Septbr. & Januar \\
\hline 3. & 13. & 29. & 14. & 8. & 28. & 14. & 28. & 18. \\
\hline 3,5 & 2,2 & 1,9 & 1,2 & 2,9 & 1,6 & 7,5 & $\begin{array}{c}\text { zwischen } \\
\text { Kraut }\end{array}$ & \\
\hline 7000 & 10500 & 65000 & 41250 & 153250 & 124000 & - & - & 195000 \\
\hline - & - & 7500 & - & v. & 1500 & - & - & 一 \\
\hline 一 & & 1000 & - & - & - & - & - & - \\
\hline- & häufig & & 1 & - & - & - & - & - \\
\hline 45000 & 1 & 1000 & 100 & - & - & - & - & - \\
\hline$\overline{150}$ & - & - & 1 & - & - & - & - & $\overline{1} 00$ \\
\hline 150 & 50 & 500 & - & - & - & - & - & 100 \\
\hline 500 & ? & 500 & - & $=$ & - & - & - & - \\
\hline - & - & 1 & 50 & 500 & 一 & - & - & 一 \\
\hline 112500 & 35000 & 1650000 & 260000 & 5500 & - & - & - & - \\
\hline 250 & E & 50 & - & - & 一 & - & - & - \\
\hline - & - & - & - & - & - & - & $\overline{2}$ & - \\
\hline 450 & - & 750 & 150 & 4 & 24 & - & - & 700 \\
\hline 300 & - & & 30 & - & - & - & - & 300 \\
\hline 500 & - & - & - & - & - & - & - & - \\
\hline 4750 & 3250 & 500 & 3750 & 1750 & 500 & - & - & 1000 \\
\hline ? & 750 & 50 & 1500 & & 250 & - & - & - \\
\hline - & - & - & - & 100 & - & - & - & - \\
\hline - & - & - & - & - & - & - & - & 一 \\
\hline$\overline{6} \overline{950}$ & - & $=$ & - & $\overline{-1}$ & $\overline{1} 000$ & - & - & $\overline{10} 00$ \\
\hline $\begin{array}{c}6250 \\
?\end{array}$ & $\begin{array}{r}10750 \\
1250\end{array}$ & $\begin{array}{r}8500 \\
500\end{array}$ & 400 & $\begin{array}{r}1750 \\
250\end{array}$ & $\begin{array}{l}1000 \\
-\end{array}$ & - & - & $\begin{array}{r}1000 \\
100\end{array}$ \\
\hline 250 & - & - & - & 100 & - & - & - & 100 \\
\hline - & - & - & - & - & - & - & - & 100 \\
\hline 500 & 600 & 100 & 150 & 350 & 7000 & - & - & 2000 \\
\hline ? & 350 & 100 & 4 & 150 & 2250 & - & - & 600 \\
\hline- & - & - & - & - & 400 & - & - & - \\
\hline- & - & - & 一 & 100 & E & E & - & - \\
\hline - & - & - & 一 & 80 & & - & - & - \\
\hline - & - & - & - & 5 & - & - & - & - \\
\hline 31000 & - & - & - & .1 & - & - & - & - \\
\hline 500 & - & - & - & - & - & - & - & $\bar{c}=0$ \\
\hline - & - & - & - & 500 & 1000 & - & - & 6500 \\
\hline - & - & - & - & 250 & 250 & - & - & 1000 \\
\hline - & - & 750 & 2000 & 100 & - & - & - & - \\
\hline$\overline{1000}$ & - & 500 & 500 & 2 & & - & - & $\overline{-1}$ \\
\hline 1000 & - & & 200 & 50 & 100 & - & - & 17000 \\
\hline - & - & $\bar{a} 00$ & $\tau_{-0}$ & $\begin{array}{r}50 \\
050\end{array}$ & 50 & - & - & - \\
\hline - & - & 1000 & $\begin{array}{l}0.00 \\
100\end{array}$ & $\begin{array}{l}200 \\
100\end{array}$ & - & - & - & - \\
\hline- & vorh. & - & 2 & 1 & $2 \check{0} 0$ & - & - & - \\
\hline
\end{tabular}




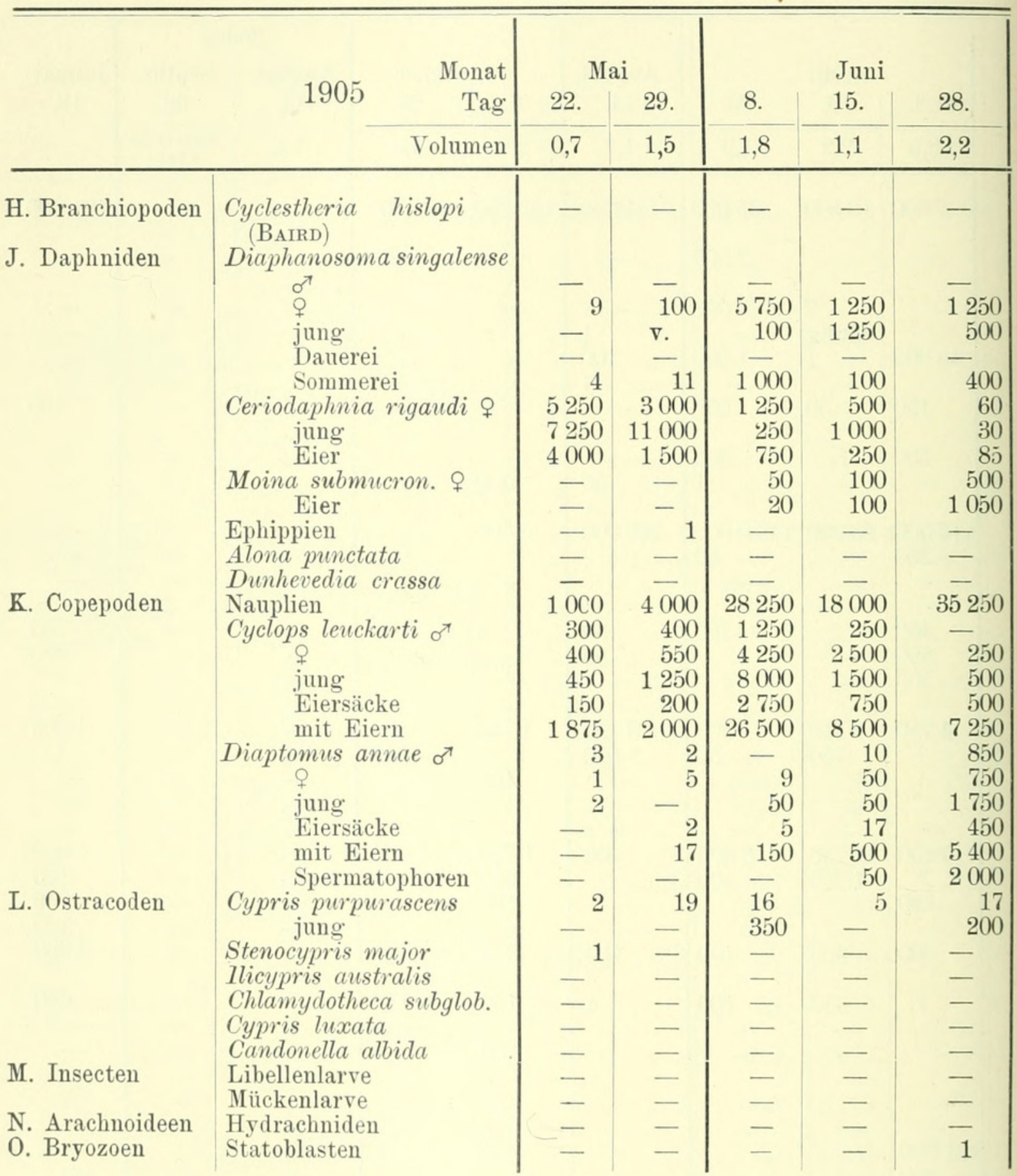




\section{h a n g.}

\begin{tabular}{|c|c|c|c|c|c|c|c|c|}
\hline \multirow[b]{3}{*}{3.} & \multirow{2}{*}{\multicolumn{2}{|c|}{ Juli }} & \multirow{3}{*}{$\begin{array}{c}\text { August } \\
14 .\end{array}$} & & & \multicolumn{2}{|c|}{ Boden } & \multirow{3}{*}{$\begin{array}{c}\text { Januar } \\
18 .\end{array}$} \\
\hline & & & & \multicolumn{2}{|c|}{ September } & August & Septbr. & \\
\hline & 13. & 29. & & 8. & 28. & 14. & & \\
\hline 3,5 & 2,2 & 1,9 & 1,2 & 2,9 & 1,6 & 7,5 & $\begin{array}{l}\text { zwischen } \\
\text { Kraut }\end{array}$ & \\
\hline - & - & 4 & - & - & - & 18 & - & - \\
\hline 750 & - & 50 & - & - & - & - & - & - \\
\hline 5000 & 600 & 2500 & 50 & 6 & 29 & - & - & 4500 \\
\hline 14500 & 1250 & 1000 & 50 & 250 & 3 & - & - & 1500 \\
\hline 60 & 150 & 50 & - & - & - & 一 & - & - \\
\hline 300 & 150 & 1000 & 100 & - & 二 & - & - & 500 \\
\hline 250 & 3250 & 1250 & 100 & - & 50 & - & - & 8000 \\
\hline 750 & 3750 & 3500 & 650 & 30 & 1250 & - & - & 8500 \\
\hline - & 750 & 500 & 20 & - & 4 & - & - & 1700 \\
\hline 450 & 100 & 250 & 100 & 1 & - & 一 & - & - \\
\hline - & - & 100 & 16 & - & - & - & 一 & 一 \\
\hline - & - & 1 & 3 & 1 & - & - & $=$ & - \\
\hline - & - & - & - & 一 & 一 & - & häufig & - \\
\hline $4 \overline{000}$ & $\overline{7000}$ & $9 \overline{500}$ & $9 \overline{000}$ & $1 \overline{750}$ & $\overline{700}$ & 一 & häutig & $135 \overline{0} 00$ \\
\hline $\begin{array}{r}4000 \\
250\end{array}$ & 500 & $\begin{array}{r}2500 \\
500\end{array}$ & $\begin{array}{r}800 \\
8\end{array}$ & $\begin{array}{r}1.00 \\
20\end{array}$ & 1 & - & - & $\begin{array}{r}155000 \\
1700\end{array}$ \\
\hline 1000 & 750 & 500 & 50 & 2 & 150 & - & - & 2500 \\
\hline 4750 & 750 & 1250 & 200 & 50 & 400 & - & - & 8000 \\
\hline 500 & 400 & 200 & 一 & - & - & - & 一 & 1000 \\
\hline 6250 & 5000 & 3000 & - & - & - & - & - & 10500 \\
\hline 250 & 3000 & 200 & 24 & 30 & 2 & - & - & - \\
\hline 750 & 3000 & 200 & 40 & 100 & 7 & - & - & - \\
\hline 500 & 250 & 750 & 300 & 500 & 4 & - & - & - \\
\hline 150 & 550 & 200 & 20 & 3 & - & - & - & - \\
\hline 1250 & 4050 & 1550 & 156 & 28 & - & - & - & - \\
\hline- & 250 & $=$ & 16 & - & - & - & - & - \\
\hline 22 & 2 & 50 & 5 & $\overline{a r c}$ & 5 & 12 & - & 300 \\
\hline 150 & - & 1000 & 50 & 250 & - & - & - & 2000 \\
\hline - & - & - & - & - & - & 3 & 6 & - \\
\hline - & - & 1 & - & - & - & 13 & $\overline{30}$ & - \\
\hline - & - & Z & - & - & - & $\overline{10}$ & 3 & - \\
\hline - & - & - & - & - & - & - & 1000 & - \\
\hline - & - & - & - & - & 一 & - & 1 & - \\
\hline$-{ }_{2}$ & - & 4 & 6 & 3 & - & häufig & mehrere & - \\
\hline-5 & -12 & $-^{2}$ & $\overline{-}$ & - & - & 1 & - & $-^{6}$ \\
\hline & & & 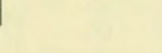 & & 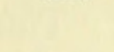 & & 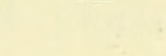 & 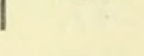 \\
\hline
\end{tabular}




\section{Literaturverzeichnis.}

1. Administration Reports, 1904, Ceylon, Part 4, Meteorology.

2. Seymons, in: Meteorol. Magazine, 1905/6, No. 477-482.

3. Apstein, Das Süsswasserplankton. 1896.

4. v. DADAY, Mikroskopische Süsswassertiere aus Ceylon. Anhangshefte zu Vol. 21 (1898) der Természetrajzi Füzetek. Budapest 1898.

5. Brady, Notes on Entomostraca collected by HALY in Ceylon, in: J. Linn. Soc. London Zool., Vol. 19, 1886.

6. Vavra, Die Süsswasser-Ostracoden Deutsch-Ostafrikas, in: DeutschOstafrika, Vol. 4, Wirbellose Tiere. 1897.

7. Hudson and Gosse, The Rotifera or Wheel Animalcules.

8. v. DADAY, Mikroskopische Süsswassertiere aus Kleinasien, in: Ber. Akad. Wiss. Wien, math.-nat. Kl., Vol. 112, Abt. 1. 1903.

9. Ehrenberg, Die Infusionsthierchen als vollkommene Organismen. Leipzig 1838.

10. v. Daday, Mikroskopische Süsswassertiere aus Deutsch Neu-Guinea, in: Természetrajzi Füzetek, Vol. 24. 1901.

11. Poppe und MrázeK, Entomostraken des Naturhist. Museums in Hamburg. 3. Die von Herrn Dr. H. Driesch auf Ceylon gesammelten Süsswasser-Entomostraken. Beiheft zu: Jahrb. Hamb. wiss. Anst., Vol. 12. 1895.

12. SARS, On some freshwater Ostracoda and Copepoda, raised from dried Australian mud, in: Forh. Vidensk. Selsk. Christiania, Aar 1889. Christiania 1890.

13. Giessbrecht und Schmen, Copepoda, I. Gymnoplea, in: Tierreich, Lief. 6. 1898.

14. SARs, On some freshwater Ostracoda, in: Forh. Vid. Selsk. Christiania, 1889, No. 8.

15. Vavra, Die Süsswasserostracoden Deutsch-Ostafrikas, in: DeutschOstafrika, Vol. 4, Wirbellose Tiere, 1897.

16. Bertram, Beiträge zur Kenntnis der Sarcosporidien nebst einem Anhange über parasitische Schläuche in der Leibeshöhle von Rotatorien, in: Zool. Jahrb., Vol. 5, Anat. 1892.

17. Labbé, Sporozoa, in: Tierreich, Lief. 5. Berlin 1899.

18. Barrois et v. DADAY, Rotifères, in: Rés. sc. d'un voyage en Palestine et en Syrie.

19. ${ }^{1}$ ) WeBer, Rotateurs, in: Zoolog. Jahrb., Vol. 24, Heft 3, Syst., 1906.

1) Diese Arbeit erschien während des Drucks meines Artikels, sodaB ich sie nur während der Korrektur benutzen konnte. 


\section{$2 \mathrm{BHL}$ Biodiversity Heritage Library}

1907. "Das Plancton im Colombo-See auf Ceylon." Zoologische Jahrbücher 25, 201-244. https://doi.org/10.5962/bhl.part.11957.

View This Item Online: https://www.biodiversitylibrary.org/item/38161

DOI: https://doi.org/10.5962/bhl.part.11957

Permalink: https://www.biodiversitylibrary.org/partpdf/11957

\section{Holding Institution}

MBLWHOI Library

\section{Sponsored by}

MBLWHOI Library

\section{Copyright \& Reuse}

Copyright Status: NOT_IN_COPYRIGHT

This document was created from content at the Biodiversity Heritage Library, the world's largest open access digital library for biodiversity literature and archives. Visit BHL at https://www.biodiversitylibrary.org. 